\title{
APPLICATIONS OF BUNDLE MAP THEORY $\left({ }^{1}\right)$
}

\author{
BY
}

\section{DANIEL HENRY GOTTLIEB}

\begin{abstract}
This paper observes that the space of principal bundle maps into the universal bundle is contractible. This fact is added to I. M. James'Bundle map theory which is slightly generalized here. Then applications yield new results about actions on manifolds, the evaluation map, evaluation subgroups of classifying spaces of topological groups, vector bundle injections, the Wang exact sequence, and $H$-spaces.
\end{abstract}

1. Introduction. Let $E \rightarrow^{p} B$ be a principal $G$-bundle and let $E_{G} \stackrel{p}{p}^{G} B_{G}$ be the universal principal $G$-bundle. The author observes that the space of principal bundle maps from $E$ to $E_{G}$ is "essentially" contractible. The purpose of this paper is to draw some consequences of that fact. These consequences give new results about characteristic classes and actions on manifolds, evaluation subgroups of classifying spaces, vector bundle injections, the evaluation map and homology, a "dual" exact sequence to the Wang exact sequence, and $H$-spaces.

The most interesting consequence is Theorem (8.13). Let $G$ be a group of homeomorphisms of a compact, closed, orientable topological manifold $M$ and let $\omega: G \rightarrow M$ be the evaluation at a base point $x_{0} \in M$. Then, if $\chi(M)$ denotes the Euler-Poincaré number of $M$, we have that $\chi(M) \omega^{*}$ is trivial on cohomology. This result follows from the simple relationship between group actions and characteristic classes (Theorem (8.8)).

We also find out information about evaluation subgroups of classifying space of topological groups. In some cases, this leads to explicit computations, for example, of $G_{1}\left(B O_{n}\right)$. See Theorem (7.3).

Then we devote ourselves to vector bundles and characterize the space of vector bundle monomorphisms into the universal bundle over the appropriate Grassmannian space as the space of mappings of the base space of the vector bundle into another Grassmannian. See Theorem (9.2).

Next we study the homomorphism which arises in the Wang exact sequence. Using bundle map theory we obtain a "dual" exact sequence which we call the Gnaw exact sequence. See Theorem (11.4). We compare this exact sequence with

Received by the editors August 16, 1971.

AMS 1969 subject classifications. Primary 5550, 5730, 5732; Secondary 5540, 5560.

Key words and phrases. Fibre bundle map, evaluation subgroup, characteristic classes of manifolds, evaluation map, Wang exact sequence, $H$-spaces.

(1) The preparation of this paper was sponsored in part by the National Science Foundation, Grant No. GP-5950.

Copyright $\odot$ 1972, American Mathematical Society 
one of Thom's and discover some mysterious facts about $H$-spaces. See Theorem (12.2). Finally, using the Gnaw exact sequence, we make some improvements in the proofs and results of [8]. See Theorem (13.2).

$\$ 2$ contains preliminaries about the topology of mapping spaces. In $\$ \S 3,4$, 5, 6 we develop the theory of bundle mappings. We show the relationships between the various types of bundle mapping spaces. In $\$ 7$, we investigate the relationship between the center of a topological group and evaluation subgroups of classifying spaces. In $\$ 8$, the study of the homology tangent bundle yields Theorem (8.13) mentioned above. In $\$ 9$ we turn our attention to the study of vector bundle monomorphisms.

The last sections of the paper are devoted to the Gnaw exact sequence and its consequences. In $\$ 10$ we review pertinent facts about the Wang homomorphisms. In $\S 11$, we exhibit the Gnaw exact sequence. In $\S 12$ we compare the Gnaw exact sequence with one of Thom's and obtain new results about $H$-spaces. In $\$ 13$ we find a general condition for evaluation subgroups to be finite.

$A$ list of sections and titles follows.

1. Introduction

PART I. Bundle map theory

$\S 2$. Preliminaries

$\S 3$. The space of principal bundle maps

$\S 4$. The space of principal bundle equivalences

$\$$ 5. Universal bundles

\$. Fibre bundles with structural group $G$

PART II. Applications of bundle map theory

$\$ 7$. Evaluation subgroups of classifying spaces

$\$$ 8. Characteristic classes and actions on manifolds

$\$ 9$. Vector bundle monomorphisms

PART III. The Gnaw exact sequence

\$10. The Wang homomorphism

$\$ 11$. The Gnaw exact sequence

$\$ 12$. Exact sequences of Thom and the Gnaw exact sequence

$\$ 13$. Some notes on [8]

\section{PART I. BUNDLE MAP THEORY}

Since I have presumed to give the body of results described below the name "bundle map theory", I should describe, to the best of my knowledge, the history of this theory.

Some of the results described appear to be folk theorems. This refers to the fibration theorems. In 1961, T. E. Stewart [18] used some special cases of these results, but he neither proved them nor gave references. In 1963, I. M. James [10] 
wrote down these theorems (most of $\$ 3$ and $\S 4$ ). He then applied his results to Stiefal manifolds and in other ways. His work was independent of Stewart's work.

In 1968, [7], I extended James' results to the category of Hurewicz fibrations. I was primarily concerned with proving that the space of "bundle maps" into the universal fibration is essentially contractible. In $\$ 5$, it is observed that this fact is true for principal fibre bundles as well. See Theorem (5.2). I consider this a very attractive fact and $I$ hope to show in the applications that it is a useful one also.

2. Preliminaries. Let $X$ and $Y$ be topological spaces. Then $L(X, Y)$ will denote the space of maps from $X$ to $Y$ endowed with the compact open topology. We shall also use the symbol $Y^{X}$. If $f: X \rightarrow Y$ is a map, we denote by $L(X, Y ; f)$ the subspace of $L(X, Y)$ of all maps homotopic to $f$.

We call $X$ a $k$-space whenever $X$ is compactly generated (i.e. any set is closed if its intersection with every compact set is closed) and Hausdorff. Locally compact spaces are $k$-spaces, metric spaces are $k$-spaces, CW complexes are $k$ spaces, if $G$ is a topological group and a $k$-space then $E_{G}$ and $B_{G}$ are $k$-spaces, any quotient of a $k$-space is a $k$-space. If $X$ and $Y$ are $k$-spaces, $X \times Y$ need not be a $k$-space, but if $X$ is locally compact and $Y$ is a $k$-space, then $X \times Y$ is a $k$ space.

Let $\alpha: X \rightarrow Z^{Y}$ be a map. Then define $\hat{\alpha}: X \times Y \rightarrow X$ by $\hat{\alpha}(x, y)=\alpha(x)(y)$. Then $\hat{\alpha}$ continuous implies $\alpha$ is continuous. On the other hand, if $Y$ is locally compact, or if $X \times Y$ is a $k$-space, then a continuous implies $\hat{\alpha}$ is continuous. The concept of $k$-space was brought into this discussion only so that we may say that $\alpha$ is continuous if and only if $\hat{\alpha}$ is continuous. For more details, see Dugundji [5, pp. 247-265].

We shall make repeated use of the relative form of the first covering homotopy property [16].

Theorem (2.1). (The relative first covering bomotopy theorem). Let $B$ be a $\mathrm{CW}$ complex. Let $E \rightarrow^{p} B$ and $E^{\prime} \rightarrow^{p^{\prime}} B^{\prime}$ be bundles with fibre $F$ and group $G$ and let $H \rightarrow K$ be a subbundle of $E \rightarrow^{p} B$ such that $K$ is a subcomplex of $B$. Suppose there is a bundle map $\tilde{f}: E \rightarrow E^{\prime}$, a bomotopy of bundle maps $\tilde{g}_{t}$ : $H \rightarrow E^{\prime}$ and a bomotopy $f_{t}: B \rightarrow B^{\prime}$ sucb that (a) $\tilde{g}_{0}=\tilde{f} \mid H$; (b) the bomotopy $g_{t}$ : $K \rightarrow B^{\prime}$ induced by $\widetilde{g}_{t}$ is just $g_{t}=f_{t} \mid K$; and finally (c) $f_{0}$ is induced by $\tilde{f}: E \rightarrow E^{\prime}$. Then there exists a bomotopy of bundle maps $\tilde{f}_{t}: E \rightarrow E^{\prime}$ such that $\tilde{f}_{t} \mid H=\tilde{g}_{t}$ and $\tilde{f}_{t}$ induces $f_{t}: B \rightarrow B^{\prime}$, and $\tilde{f}=\tilde{f}_{0}$.

By [W. Huebsch, On the covering bomotopy theorem, Ann. of Math. 61 (1955), 555-563], the theorem above requires that $B$ be normal and paracompact. Since by a theorem of Mitsuru Tsuda, every CW complex is paracompact, the hypothesis is satisfied. This eliminates the need for James' condition of a locally finite CW complex which he uses in [10]. 
3. The space of principal bundle maps. We shall concern ourselves with principal bundles $E \rightarrow^{p} B$. The fibre is a topological group of homeomorphisms of $E$ which operates on the right of $E$ such that the following diagram commutes

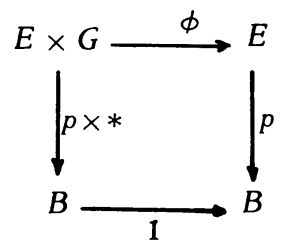

We denote $\phi(e, g)$ by $e \cdot g$.

If we have two principal bundles with the same group $G, E \rightarrow^{p} B$ and $E^{\prime}$ $\rightarrow^{p^{\prime}} B^{\prime}$, then a map $\tilde{f}: E \rightarrow E^{\prime}$, which is a fibre preserving map that is a homeomorphism from each fibre onto its image such that $\tilde{f}(e \cdot g)=\tilde{f}(e) \cdot g$ for all $e \epsilon$ $E$ and $g \in G$, is known as a principal bundle map.

Now we let $L^{*}\left(E, E^{\prime}\right)$ represent the space of all principal bundle maps from $E$ to $E^{\prime}$ endowed with the compact open topology. Let $L^{*}\left(E, E^{\prime} ; \tilde{f}\right)$ be the path component of $\tilde{f}$. Now every principal bundle map $\tilde{f}: E \rightarrow E^{\prime}$ induces a map of the base spaces, $f: B \rightarrow B^{\prime}$. Let $\Phi: L^{*}\left(E, E^{\prime} ; \tilde{f}\right) \rightarrow L\left(B, B^{\prime} ; f\right)$ be the function which sends every bundle map into the induced map on the base spaces. Here $L\left(B, B^{\prime} ; f\right)$ is the space of maps from $B$ to $B^{\prime}$ homotopic to $f$ endowed with the compact open topology.

Proposition (3.1). With the notation above, $\Phi: L^{*}\left(E, E^{\prime} ; \tilde{f}\right) \rightarrow L\left(B, B^{\prime} ; f\right)$ is a Serre fibration.

Proof. Very similar to proof of Theorem (2.1) in [10].

The fibre of $\Phi$ over a map $f: B \rightarrow B^{\prime}$ will be denoted by $L^{* *}\left(E, E^{\prime}\right)$. This is the set of all bundle maps which cover a given base map $f$.

Suppose we have three principal $G$-bundles whose total spaces are $E, E^{\prime}$ and $E^{\prime \prime}$ and whose base spaces are $B, B^{\prime}$ and $B^{\prime \prime}$ respectively. Suppose they are related by two principal bundle maps $E \rightarrow^{\prime} E^{\prime} \rightarrow_{\widetilde{g}} E^{\prime \prime}$. Then composition on the left by $\widetilde{g}$ gives rise to a map $\widetilde{g}_{\#}: L^{*}\left(E, E^{\prime} ; \tilde{f}\right) \rightarrow L^{*}\left(E, E^{\prime \prime} ; \widetilde{g} \circ \tilde{f}\right)$. Observe that $\widetilde{g}_{\#}$ is a fibre preserving map, that is, the following diagram commutes:

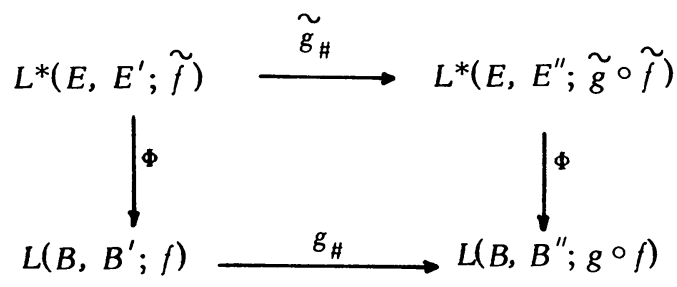

where $f$ and $g$ are induced by $\tilde{f}$ and $\widetilde{g}$ and $g_{\#}$ is composition on the left.

On the other hand, composition on the right by $\tilde{f}$ gives a map $\tilde{f}^{\#}: L^{*}\left(E^{\prime}, E^{\prime \prime}\right)$ 
$\rightarrow L^{*}\left(E, E^{\prime \prime}\right)$ which is fibre preserving, that is, the following diagram commutes:

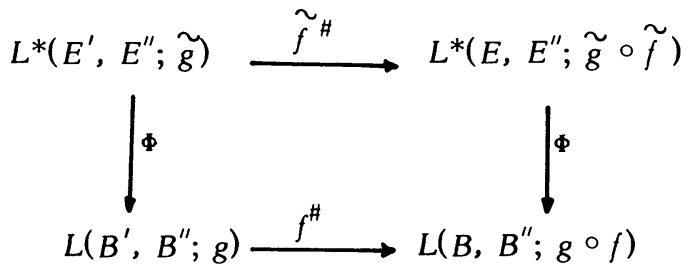

An important special case of (3.3) occurs when $\tilde{f}: E \rightarrow E^{\prime}$ is an inclusion map. Thus $E \rightarrow^{p} B$ is the restriction of $E^{\prime} \rightarrow^{p \prime} B^{\prime}$ to the bundle over $B \subset B^{\prime}$. Thus $E \subset E^{\prime}$ and $\tilde{f}^{\#}$ is just the restriction $\rho$ of any principal bundle map $\tilde{\alpha}: E^{\prime}$ $\rightarrow E^{\prime \prime}$ to $\rho(\tilde{\alpha}): E \rightarrow E^{\prime \prime}$.

Proposition (3.4). With above notation, assume that $B$ is a subcomplex of the $\mathrm{CW}$ complex $B^{\prime}$. Then $\rho: L^{*}\left(E^{\prime}, E^{\prime \prime}\right) \rightarrow L^{*}\left(E, E^{\prime \prime}\right)$ is a Serre fibration.

Proof. This theorem is essentially Theorem (2.3) of I. M. James [10]. It depends on the relative version of the first covering homotopy theorem.

The fibre of $\rho: L^{*}\left(E^{\prime}, E^{\prime \prime}, \widetilde{g}\right) \rightarrow L^{*}\left(E, E^{\prime \prime} ; \rho(\widetilde{g})\right)$ over $\rho(\widetilde{g})$ will be written $L_{B}^{* *}\left(E^{\prime}, E^{\prime \prime}\right)$ and is the subspace of all bundle maps $E^{\prime} \rightarrow E^{\prime \prime}$ whose restriction to $E$ is equal to $\rho(\widetilde{g})$, the restriction of $\widetilde{g}$ to $E$.

An important example of the restriction map occurs when we let $B=*$, the base point of $B^{\prime}$. Then $E=G$, the group. Now $L^{*}\left(G, E^{\prime \prime}\right)$ is homomorphic to $E^{\prime \prime}$ since any bundle map from $G \rightarrow E^{\prime \prime}$ is determined by its value on the identity element $e \in G$. Similarly, $L\left(*, B^{\prime \prime}\right)$ is just $B^{\prime \prime}$ and so we get a fibre preserving map from diagram (3.3),

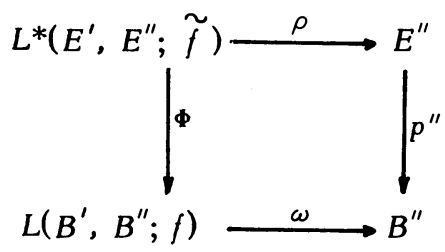

Here $\omega$ is the evaluation map.

This observation was made and used by I. M. James, (2.4) of [10]. We shall use it in $\$ 7$.

4. The space of principal bundle equivalences. A principal bundle equivalence $f: E \rightarrow E^{\prime}$ is a principal bundle map which is also a homeomorphism and induces the identity map on the base spaces. We shall let $L^{* *}(E, E)$ denote the space of self bundle equivalences. We shall show that $L^{* *}(E, E)$ is homeomorphic to the fibre of $\Phi: L^{*}\left(E, E^{\prime}\right) \rightarrow L\left(B, B^{\prime}\right)$.

Let $k: X \rightarrow B$ be any map. Then the induced principal bundle $k^{*}(E) \rightarrow^{k(p)} X$ is defined by $k^{*}(E)=\{(x, e) \in X \times E \mid k(x)=p(e)\}$ and the projection $k(p)$ is given by $(x, e) \mapsto x$. 
We define the natural map $\eta: k^{*}(E) \rightarrow E$ by $\eta(x, e)=e$. We have the commutative diagram

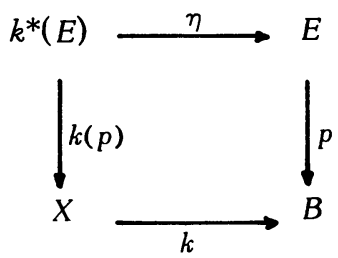

Clearly $\eta$ is a principal bundle map.

The importance of $\eta$ comes from the fact that any principal bundle map $\tilde{f}: E$ $\rightarrow E^{\prime}$ must factor through $\eta$. In fact we have $\tilde{f}=\eta \circ \bar{f}$ where

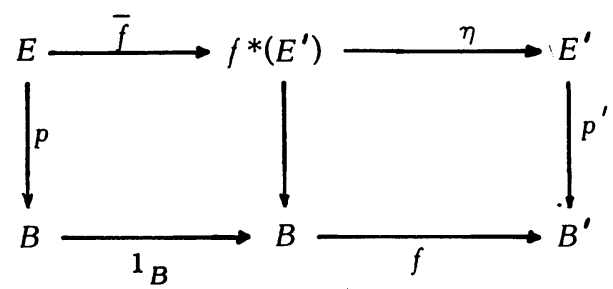

where $\bar{f}$ is the principal bundle equivalence given by $\bar{f}(\dot{e})=(p(e), \tilde{f}(e))$.

Now the correspondence $\bar{g} \circ \eta \mapsto \bar{g}$ shows that $L^{* *}\left(E, E^{\prime}\right)$ is homeomorphic to $L^{* *}\left(E, f^{*}\left(E^{\prime}\right)\right)$ and the correspondence $\tilde{b} \mapsto \bar{f} \circ \widetilde{b}$ shows that $L^{* *}\left(E, f^{*}\left(E^{\prime}\right)\right)$ is homeomorphic to $L^{* *}(E, E)$. This gives us the following proposition.

Proposition (4.3). $L^{* *}(E, E)$ is bomeomorphic to $L^{* *}\left(E, E^{\prime}\right)$.

It is possible to characterize $L^{* *}$ as follows. We denote by $\xi_{G}$ the function space of maps $u: E \rightarrow G$ which satisfy the condition

$$
u(e \cdot g)=g^{-1} u(e) g \quad(e \in E, g \in G) .
$$

If $\tilde{f}: E \rightarrow E^{\prime}$ is a bundle map then so is $\tilde{f} \cdot u$, where $u \in \xi_{G}$ and $(\tilde{f} \cdot u)(e)=$ $\tilde{f}(e) \cdot u(e)$. Now $\Phi(\tilde{f})=\Phi(\tilde{f} \cdot u)$, so the correspondence $u \mapsto \tilde{f} \cdot u$ is a map from $\xi_{G} \rightarrow L^{* *}\left(E, E^{\prime}\right)$. This correspondence is a homeomorphism. Thus $L^{* *}(E, E)$ is homeomorphic to $\xi_{G}$. Thus, if $G$ is finite, $L^{* *}$ is finite; and if $G$ is commutative, then $L^{* *}$ is homeomorphic to $L(B, G)$. See I.M. James, Theorem (2.2), in [10].

5. Universal bundles. So far, bundle map theory was essentially known to James. In this section we introduce the key observation, which is Theorem (5.2), that the space of bundle maps into a universal bundle is essentially contractible.

For any topological group $G$, a principal $G$-bundle $E_{G} \stackrel{p}{p} G_{B}$ is called 
universal if the homotopy groups of $E_{G}$ are trivial. Universal bundles have the property that any principal bundle $E \rightarrow^{p} B$ over a $C W$ complex $B$ with the same group $G$ is principal bundle equivalent to an induced bundle $k^{*}\left(E_{G}\right)$ induced by a map $k: B \rightarrow B_{G}$. In addition, $k$ is called a classifying map for $E \rightarrow^{p} B$ and any two classifying maps are homotopic. Milnor [11] has shown a way to construct universal principal bundles for any topological group $G$.

The key observation comes from the following.

Lemma (5.1). If $f$ and $g: E \rightarrow E_{G}$ are two principal bundle maps, then $f$ is bomotopic to $g$ by a bomotopy of principal bundle maps (written $f \simeq g$ ). (We are assuming that $B$ is a $\mathrm{CW}$ complex.)

Proof. This is easily shown to be true. Let $f: E \rightarrow E$ be a principal fibre bundle equivalence and define the mapping torus

$$
E_{f}=\{(e, t) \mid(e, t) \in E \times I \text { and }(e, 1) \equiv(f(e), 0)\} .
$$

Let $q_{f}: E_{f} \rightarrow B \times S^{1}$ be defined by $q_{f}(e, t)=(p(e), t)$. Now $q_{f}$ is well defined since $p(f(e))=p(e)$. It is clear that $q_{f}$ is a fibre bundle map. The only question that remains is whether $q_{f}: E_{f} \rightarrow B \times S^{1}$ is a principal $G$-bundle.

Let $\psi: E \times G \rightarrow E$ be the action of $G$ on $E$. We define $\psi_{f}: E_{f} \times G \rightarrow E_{f}$ by $\psi_{f}((e, t), g)=(\psi(e, g), t)$. Since $f$ is a principal bundle equivalence and thus commutes with the action of $G$, we have

$$
\begin{aligned}
\psi_{f}((e, 1), g) & =(\psi(e, g), 1) \equiv(f \psi(e, g), 0) \\
& =(\psi(f(e), g), 0)=\psi_{f}((f(e), 0), g) .
\end{aligned}
$$

So $\psi_{f}$ is well defined and so $q_{f}$ is a principal $G$-fibration.

The existence of the mapping torus fibration allows us to conclude that there is a principal bundle map $\tilde{s}: E \rightarrow E_{G}$ such that $\widetilde{s}$ is homotopic, through principal bundle maps, to $\widetilde{s} \circ f$.

Now we must show that there is one such $\widetilde{s}$ for all homotopy equivalences $f$ : Each $E_{f}$ contains a canonical $E \subset E_{f}$. Say $E=E \times 0$ in $E_{f}$. Then define $M$ to be the union of all the $E$ identified at $E$. Then $M \rightarrow^{q^{\prime}} B \times\left(\bigvee S^{1}\right)$, defined by $q^{\prime}(e)$ $=q_{f}(e)$ if $e \in E_{f}$, is a principal fibration and hence there exists a principal bundle $\operatorname{map}_{\sim} \tilde{r}: M \rightarrow E_{\sim}$. Let $E \subset M$ be the identified subspace of all the $E_{f}$ 's. Define $\tilde{s}$ $=\tilde{r} \mid E$. Now $\tilde{r}$ restricted to an appropriate $E_{f}$ gives rise to the bundle map homotopy $E \rightarrow^{i_{t}} E_{f} \rightarrow^{\widetilde{r}} E_{G}$ (where $i_{t}(e)=(e, t) \in E_{f}$ ) so that $\widetilde{r} \circ i_{0}=\widetilde{s}$ and $\widetilde{r} \circ i_{1}=\widetilde{s} \circ f$. We have just shown that there exists a principal bundle map $\tilde{s}: E \rightarrow E_{G}$ such that for any principal bundle equivalence $f: E \rightarrow E$ we have $\widetilde{s} \simeq \widetilde{s} \circ f$.

Now let $\widetilde{g}: E \rightarrow E_{G}$ be a principal bundle map. We shall show $\widetilde{g} \simeq \widetilde{s}$. Since this holds for arbitrary $\tilde{g}$, the lemma will be proved.

Let $g$ and $s: B \rightarrow B_{G}$ be induced by $\widetilde{g}$ and $\widetilde{s}$ respectively. Then both $g$ and 
$s$ are classifying maps for $E \rightarrow^{p} B$, so $g$ is homotopic to $s$. So, by the homotopy covering property, we may assume that $g=s$. Then we obtain the following diagram (see (4.2)):

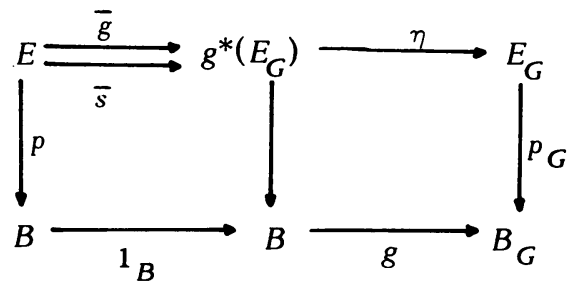

Here $\bar{g}$ and $\bar{s}$ are principal bundle equivalences.

Now $\bar{s}^{-1} \circ \bar{g}: E \rightarrow E$ is a self principal bundle equivalence. Now by the third paragraph above, we have

$$
\tilde{s} \simeq \tilde{s} \circ\left(\bar{s}^{-1} \circ \bar{g}\right) \simeq(\eta \circ \bar{s}) \circ\left(\bar{s}^{-1} \circ \bar{g}\right) \simeq \eta \circ \bar{g}=\tilde{g} .
$$

This proves the lemma.

We shall digress to introduce the concept of "essentially homotopic". We say that $f$ and $g: X \rightarrow Y$ are essentially bomotopic if, for every map $s: p \rightarrow X$ from a compact polyhedron $P$ to $X$, we have $f \circ s$ homotopic to $g \circ s$. It is easy to see that two essentially homotopic maps induce the same homomorphisms on homotopy groups and on singular homology and cohomology.

If the identity map $1_{X}: X \rightarrow X$ is essentially homotopic to a constant map, we say that $X$ is essentially contractible. Then $\pi_{i}(X)=0$ for all $i$ and hence $H_{i}(X, Z)$ $=0$ for all $i$. Now we can state the main consequence of Lemma (5.1).

Theorem (5.2). $L^{*}\left(E, E_{G}\right)$ is essentially contractible.

Proof. Let $k: B \rightarrow{ }^{B}{ }_{G}$ be a classifying map for $E \rightarrow{ }^{p} B$. Let $\tilde{k}: E \rightarrow E_{G}$ be a principal bundle map which induces $k$. Let $i: X \rightarrow C X$ be the obvious inclusion of $X$ into the cone of $X$. Then we have the commutative diagram of principal bundles.

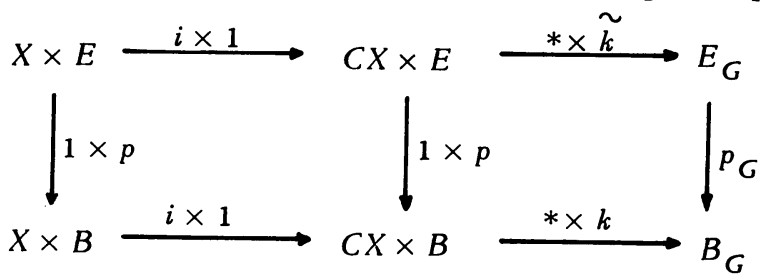

Now let $X$ be a compact CW complex. Then any map $\alpha: X \rightarrow L^{*}\left(E, E_{G}\right)$ gives rise to an associated principal bundle map

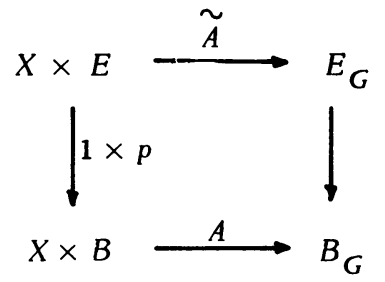


Now by Lemma (5.1), $\tilde{A} \widetilde{\simeq}(* \times \widetilde{k}) \circ(i \times 1)$. Hence the associated map $\alpha: X$ $\rightarrow L^{*}\left(E, E_{G}\right)$ factors through $C X$. Hence $\alpha$ is homotopic to a constant.

Now we extend our theory to the case of principal bundle maps which restrict to a given principal bundle map on a subbundle. We suppose $K \subset B$ is a subcomplex of $B$. Then the principal $G$-bundle $E \rightarrow^{p} B$ restricts to a principal $G$-bundle over $K$, written $p^{-1}(K) \rightarrow K$. Let $\tilde{g}: p^{-1}(K) \rightarrow E^{\prime}$ be a principal bundle map. Let $L_{K}^{*}\left(E, E^{\prime} ; \tilde{g}\right)$ be the space of all principal bundle maps from $E$ to $E^{\prime}$ which restrict on $p^{-1}(K)$ to $\tilde{g}$. As noted before, this is the fibre of $L^{*}\left(E, E^{\prime}\right) \rightarrow^{\Phi}$ $L^{*}\left(p^{-1}(K), E^{\prime}\right)$.

Proposition (5.3). $L_{K}^{*}\left(E, E^{\prime} ; \tilde{g}\right) \rightarrow^{\Phi} L_{K}\left(B, B^{\prime} ; f\right)$ is a Serre fibration such that $\Phi(\tilde{f})=f$ and $\rho(\tilde{f})=\tilde{g}$, where $\tilde{f}$ is some element of $L_{K}^{*}\left(E, E^{\prime} ; \tilde{g}\right)$.

Proof. This follows from the relative first covering homotopy theorem.

In fact we obtain the following commutative diagram where each row or column is a Serre fibration written in the form $F \rightarrow^{i} E \rightarrow^{p} B$ where $F$ is the fibre. We are assuming that $K$ is a subcomplex of $B$. We further assume that $\tilde{f} \mid p^{-1}(K)=$ $\widetilde{g}$ and that $f \mid K=\Phi(\widetilde{g})=g$. In all cases, $\rho$ means the restriction map and $i$ means inclusion.

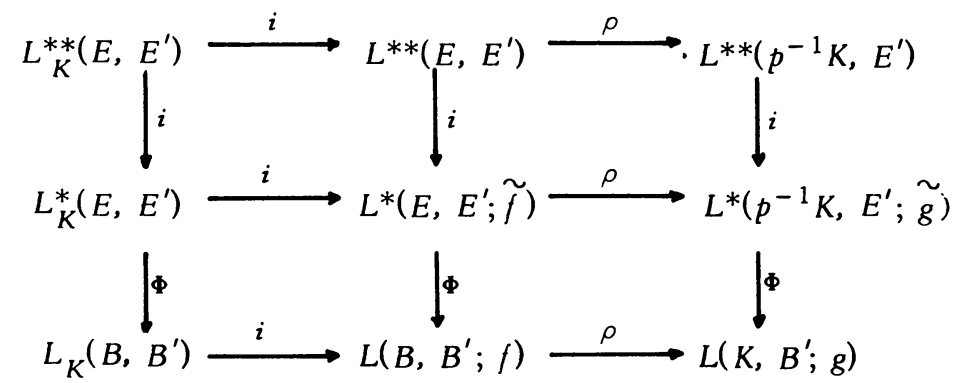

The first column is a Serre fibration by (5.3), the second and third by (3.1). The bottom row is a Serre fibration, as is well known; the middle row by Proposition (3.4); and the top row is a fibration for the same basic reason that the middle row is, namely by an application of the relative form of the first covering homotopy theorem.

Now suppose that we have two principal bundle maps $\tilde{f}$ and $\tilde{g}: E \rightarrow E_{G}$ such that $\tilde{f}\left|p^{-1}(K)=\tilde{g}\right| p^{-1}(K)$. We know that $\tilde{f}$ is homotopic to $\tilde{g}$ through principal bundle maps by (5.1). We wish to know that $\tilde{f}$ is homotopic to $\tilde{g}$ by a homotopy $\tilde{b}_{t}$ of bundle maps such that $\tilde{b}_{t}\left|p^{-1}(K)=\tilde{f}\right| p^{-1}(K)$. Again, this follows from the relative form of the first homotopy covering theorem as follows. Let $H: E \times I \rightarrow E_{G}$ be defined by $H(e, t)=\tilde{b}_{t}(e)$. Then $H$ is a principal bundle map. Consider the subbundle $E \times 0 \cup E \times 1 \cup p^{-1}(K) \times I$ of $E \times I$. We define a partial homotopy

$$
K_{s}:(E \times 0) \cup(E \times 1) \cup\left(p^{-1}(K) \times I\right) \rightarrow E_{G}, \quad s \in I,
$$


by $K_{0}=H$ restricted to the subbundle

$K_{s}(e, 0)=\tilde{f}(e)$,

$K_{s}(e, 1)=\widetilde{g}(e)$,

$K_{s}(e, t) \mid p^{-1}(K) \times I$ is a homotopy of principal bundle maps where $K_{0}=H$ and $K_{1}(e, t)=\tilde{f}(e)$.

Then there exists a homotopy of principal bundle maps $H_{s}: E \times I \rightarrow E_{G}$ such that $H_{0}=H$ and $H_{s} \mid(E \times 0) \cup(E \times 1) \cup\left(p^{-1}(K) \times I\right)=K_{s}$. Then $H_{1}$ is the required homotopy from $\tilde{f}$ to $\widetilde{g}$ such that $H_{1}(e, t)=\tilde{f}(e)=\widetilde{g}(e)$ if $e \in p^{-1}(K)$. We have just shown the following.

Lemma (5.5). If $\tilde{f}$ and $\tilde{g}: E \rightarrow E_{G}$ are principal bundle maps such that $\tilde{f}\left|p^{-1} K=\tilde{g}\right| p^{-1} K$, then $f \simeq g\left(\operatorname{rel} p^{-1} K\right)$.

This lemma gives us the following theorem in the same way that Lemma (5.1) gives Theorem (5.2).

Theorem (5.6). $L_{K}^{*}\left(E, E_{G}\right)$ is essentially contractible.

It is worthwhile observing that Theorem (5.6) gives a homotopy characterization of $L_{K}^{* *}(E, E)$. Namely, $L_{K}^{* *}$ is weak homotopy equivalent to $\Omega\left(L_{K}\left(B, B{ }_{G} ; f\right)\right)$, the loop space of the space of maps of $B \rightarrow B_{G}$ homotopic to $f$ which restrict on $K$ to $f \mid K$. Compare this with James' characterization of $L^{* *}$ in $\$ 4$.

6. Fibre bundles with structural group $G$. We shall extend the theory of bundle maps to fibre bundles with fibre $F$ and structural group $G$.

Let $E \rightarrow^{p} B$ be a principal bundle with fibre the group $G$. Suppose $G$ acts on a space $F$ on the left as a group of homeomorphisms. Consider all pairs $(e, x)$ $\epsilon E \times F$. There is an equivalence relation between these pairs given by $(e, x) \sim$ $\left(e \cdot g, g^{-1} \cdot x\right)$. Let $\langle e, x\rangle$ denote the equivalence class of $(e, x)$ under this relation. The quotient space of $E \times F$ by $\sim$ (whose points are $\langle e, x\rangle$ ) is denoted by $E \times{ }_{G} F$.

The map $\bar{p}: E \times{ }_{G} F \rightarrow B$ given by $\bar{p}(\langle e, g\rangle)=p(e)$ is a fibre bundle projection and the fibre is $F$. The fibre bundle $F \rightarrow E \times_{G} F \rightarrow \bar{p}^{\bar{p}} B$ is called an associated bundle of $E$. Every locally trivial fibre bundle with structural group $G$ is obtainable by the above process. For a good short account of these concepts, see $\$ 12$ of Borel [2].

If $\tilde{f}: E \rightarrow E^{\prime}$ is a principal bundle map, we get an induced map $E \times_{G} F \rightarrow{ }^{\bar{f}}$ $E^{\prime} \times_{G} F$ given by $\bar{f}(\langle e, x\rangle)=\langle\tilde{f}(e), x\rangle$.

Now let $E$ and $E^{\prime}$ be total spaces of fibre bundles with fibre $F$ and group $G$. We say a map $\bar{f}: E \rightarrow E^{\prime}$ is a bundle map if $\bar{f}$ is induced by a principal bundle map between the associated principal bundles.

Now let $E$ and $E^{\prime}$ be fibre bundles with fibre $F$ and group $G$. Then we define the space, $L^{*}\left(E, E^{\prime}\right)$, of bundle maps. 
The space of bundle maps is related to the space of principal bundle maps as follows. Let $E$ and $E^{\prime}$ be principal bundles. Then let $\varphi: L^{*}\left(E, E^{\prime}\right) \rightarrow$ $L^{*}\left(E \times_{G} F, E^{\prime} \times_{G} F\right)$ be the map which takes principal bundle maps into their induced bundle maps, $\varphi(\tilde{f})=\bar{f}$. Note $\varphi$ is continuous and onto.

Proposition (6.1). Suppose $G$ acts effectively on $F$. Then $\varphi: L^{*}\left(E, E^{\prime}\right) \rightarrow$ $L^{*}\left(E \times_{G} F, E^{\prime} \times_{G} F\right)$ is a bomeomorphism.

Proof. Suppose $\tilde{f}$ and $\tilde{g} \in L^{*}\left(E, E^{\prime}\right)$ are not equal. We may assume that they induce the same base map. Then there exists an $e_{0} \in E$ such that $\tilde{f}\left(e_{0}\right) \neq \tilde{g}\left(e_{0}\right)$. Thus there is some $g \in G$ not equal to the identity such that $\tilde{f}\left(e_{0}\right) \cdot g=\widetilde{g}\left(e_{0}\right)$. Since $G$ acts effectively on $F$, there is an $x_{0} \in F$ such that $g \cdot x_{0} \neq x_{0}$. Then consider the induced maps $\bar{f}=\varphi(\tilde{f})$ and $\bar{g}=\varphi(\tilde{g})$. Then

$$
\bar{f}\left(\left\langle e_{0}, x_{0}\right\rangle\right)=\left\langle\tilde{f}\left(e_{0}\right), x_{0}\right\rangle
$$

and

$$
\bar{g}\left(\left\langle e_{0}, x_{0}\right\rangle\right)=\left\langle\tilde{g}\left(e_{0}\right), x_{0}\right\rangle=\left\langle\tilde{f}\left(e_{0}\right) \cdot g, x_{0}\right\rangle=\left\langle\tilde{f}\left(e_{0}\right), g^{-1} \cdot x_{0}\right\rangle .
$$

Since $x_{0} \neq g \cdot x_{0}$, we have $\bar{f} \neq \bar{g}$. Thus $\varphi$ is injective. Also $\varphi$ is open and onto. So $\varphi$ is a homeomorphism.

Because the relative form of the first covering homotopy theorem holds for fibre bundles with fibre $F$ and group $G$, we get an analogous theory as outlined in $\S 3$ and $\S 4$. For instance $L^{* *}\left(E, E^{\prime}\right) \rightarrow^{i} L^{*}\left(E, E^{\prime}\right) \rightarrow^{\Phi} L\left(B, B^{\prime}\right)$ is a Serre fibration.

Now suppose that $E_{G} \rightarrow{ }^{p} B_{G}$ is a universal principal bundle. Then $E_{G} \times{ }_{G} F \rightarrow{ }^{\bar{p}} B_{G}$ is a universal fibre bundle for fibre $F$ and structural group $G$.

Theorem (6.2). Suppose $G$ acts effectively on $F$. Suppose $E$ and $E_{\infty}$ are fibre bundles with fibre $F$ and group $G$ and suppose that $E_{\infty}$ is universal (i.e. $E_{\infty}$ $\left.=E_{G} \times_{G} F\right)$. Then $L^{*}\left(E, E_{\infty}\right)$ is essentially contractible.

Proof. Use Proposition (6.1) and Theorem (5.2).

We shall consider fibre bundles with fibre a manifold $M$ and group $G(M)$, the group of homeomorphisms. Also we shall consider vector bundles in the sequel.

\section{PART II. APPLICATIONS OF BUNDLE MAP THEORY}

7. Evaluation subgroups of classifying spaces. We shall begin this section by noting Milnor's construction of the universal principal bundle $E_{G} \rightarrow B_{G}$, [11]. We shall observe that $G$ operates on $E_{G}$ as a group of principal bundle maps and the center of $G$ operates as a group of principal bundle equivalences. By using the fact that $L^{*}$ is essentially contractible, we obtain the result that the evaluation subgroups must contain naturally defined groups based on the center of $G$. 
Thus, in some cases, we can actually find the evaluation subgroups of $B_{G}$.

We consider the infinite join of the group $G$, given by $G * G * \ldots$. The points of $E_{G}=G * G * \ldots$ will be represented as an infinite sequence $t_{1} g_{1} * t_{2} g_{2} * \ldots$ where $g_{i} \in G$ and $t_{i} \in I$ and $\Sigma t_{i}=1$ and all but a finite number of the $t_{i}$ are 0 . We identify $0 \cdot g$ with 0 for all $g \in G$. Thus $t_{1} g_{1} * t_{2} g_{2} * \ldots * 0 * \cdots$ has 0 's in all but a finite number of places. If $E_{G}$ is given the suitable topology, then $E_{G}$ is contractible.

Now $G$ operates on the right of $E_{G}$ by

$$
\left(t_{1} g_{1} * t_{2} g_{7} * \cdots\right) g=t_{1}\left(g_{1} g\right) * t_{2}\left(g_{2} g\right) * \cdots \text {. }
$$

Any two points $e$ and $e^{\prime} \in E_{G}$ are said to be equivalent if there exists a $g \in G$ such that $e=e^{\prime} g$. The equivalence class of $t_{1} g_{1} * t_{2} g_{2} * \ldots$ will be denoted by $\left\langle t_{1} g_{1} * t_{2} g_{2} * \cdots\right\rangle$. The quotient space $E_{G} / G$ is denoted $B_{G}$ and consists of points $\left\langle t_{1} g_{1} * t_{2} g_{2} * \cdots\right\rangle$. The projection $p$ which sends $t_{1} g_{1} * t_{2} g_{2} * \cdots \rightarrow$ $\left\langle t_{1} g_{1} * t_{2} g_{2} * \cdots\right\rangle$ gives a principal bundle $G \rightarrow E_{G} \rightarrow^{p} B_{G}$ which is universal.

Now, note that $G$ acts on the left of $E_{G}$. The action is given by

$$
g\left(t_{1} g_{1} * t_{2} g_{2} * \cdots\right)=t_{1}\left(g g_{1}\right) * t_{2}\left(g g_{2}\right) * \cdots .
$$

Let $l_{g}: E_{G} \rightarrow E_{G}$ be given by $l_{g}(e)=g \cdot e$. Note that $l_{g}\left(e g^{\prime}\right)=l_{g}(e) g^{\prime}$. Thus $l_{g}$ is a principal bundle map from $E_{G} \rightarrow E_{G}$. The correspondence given by $g \rightarrow l_{g}$ defines a canonical imbedding $j: G \rightarrow L^{*}\left(E_{G}, E_{G}\right)$.

One immediate consequence is the following fact. Let $m: G \times B_{G} \rightarrow B_{G}$ be given by

$$
m\left(g,\left\langle t_{1} g_{1} * t_{2} g_{2} * \cdots\right\rangle\right)=\left\langle t_{1}\left(g g_{1}\right) * t_{2}\left(g g_{2}\right) * \cdots\right\rangle .
$$

Proposition (7.1). The projection $G \times B_{G} \rightarrow{ }^{\pi} B_{G}$ is essentially bomotopic to $m$.

Proof. Note that $m$ is the associated map of $\phi \circ j: G \rightarrow L^{*}\left(E_{G}, E_{G}\right) \rightarrow$ $L\left(B_{G}, B_{G}\right)$. Since $L^{*}$ is essentially contractible, $\phi \circ j$ is essentially homotopic to a constant map. Thus the associated map $m$ is essentially homotopic to the associated map of a constant map, $\pi$.

Let $C$ be the center of $G$. If $c \in C$, then $l_{c}$ is a principal bundle equivalence since $l_{c}$ induces the identity on $B_{G} \rightarrow B_{G}$. In fact, the induced map operates on points of $B_{G}$ as follows:

$$
\begin{aligned}
\left\langle t_{1} g_{1} * t_{2} g_{2} * \ldots\right\rangle & \mapsto\left\langle t_{1}\left(c g_{1}\right) * t_{2}\left(c g_{2}\right) * \ldots\right\rangle \\
& =\left\langle t_{1}\left(g_{1} c\right) * t_{2}\left(g_{2} c\right) * \ldots\right\rangle \\
& =\left\langle t_{1} g_{1} * t_{2} g_{2} * \ldots\right\rangle .
\end{aligned}
$$


Hence we have the imbedding $j: C \rightarrow L^{* *}\left(E_{G}, E_{G}\right)$.

Now consider the following commutative diagram, which arises from diagram (3.5).

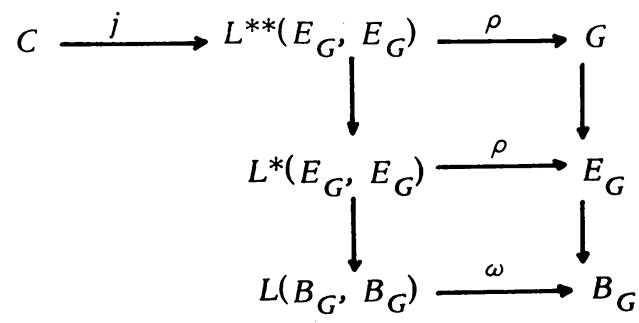

Observe that $\rho \circ j=i$, the inclusion map of $C \rightarrow^{i} G$. Let $d$ represent the boundary homomorphism from the homotopy groups of the base to the homotopy groups of the fibre. The image of $\omega_{*}: \pi_{i}\left(L\left(B_{G}, B_{G}\right)\right) \rightarrow \pi_{i}\left(B_{G}\right)$ is denoted $G_{i}\left(B_{G}\right)$ and is called the ith evaluation subgroup of $B_{G}$.

Theorem (7.3). With notation above, the image of $d^{-1} \circ i_{*}: \pi_{i}(C) \rightarrow \pi_{i+1}\left(B_{G}\right)$ is contained in $G_{i+1}\left(B_{G}\right)$ for all $i$. Note $d$ is an isomorphism.

Proof. From the fibre homotopy exact ladder arising from (7.2), we have $\rho_{*}{ }^{\circ}$ $d=d \circ \omega_{*}$. Thus the image of $d^{-1} \circ \rho_{*}$ is contained in $G_{i+1}\left(B_{G}\right)$ and hence the image of $d^{-1} \circ \rho_{*} \circ j_{*}=d^{-1} \circ i_{*}$ is.

Remark (7.4). Since $j: C \rightarrow L^{* *}$ and $\rho: L^{* *} \rightarrow G$ are both homomorphisms, one can complete diagram (7.2) to

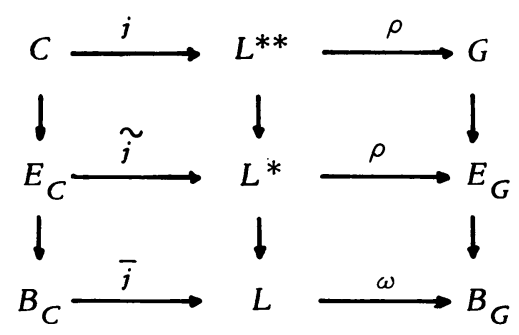

where $\tilde{j}$ and $\bar{j}$ are induced by $j$ as is usual in the theory of universal principal bundles. This map $\bar{j}$ means that $B_{C}$ operates on $B_{G}$.

Actually, there are technical flaws in the above argument, due to the fact the classifying space theory has not been developed for Serre fibrations. However, if we use the Steenrod-Milgram version of classifying bundles [17], we can see the action: In fact, the multiplication $C \times G \rightarrow^{m} G$ is actually a homomorphism since $C$ commutes with $G$. Thus $m$ induces an action $B_{C} \times B_{G} \rightarrow B_{G}$ due to the naturality of these classifying bundles and the fact that $B_{C \times G}=B_{C} \times B_{G}$. This is the appropriate action as can easily be checked. 
Now $C$ is a commutative group, hence it must be a product of EilenbergMac Lane spaces. If $G$ is compact and finite dimensional, then $C$ must be a torus, or a union of tori.

Corollary (7.5). Let $G$ be a compact finite dimensional topological group and let the center have dimension $n$. Then $G_{2}\left(B_{G}\right)$ contains a free abelian group of rank $n$.

Proof. Consider the homotopy exact sequence resulting from the fibration $C$ $\rightarrow^{i} G \rightarrow G / C$. Since $G / C$ is a finite dimensional group, $\pi_{2}(G / C)=0$ by Browder's theorem. Since $C$ is a torus of dimension $n, \pi_{1}(C)$ is a free abelian group of dimension $n$. Thus $\iota_{*}: \pi_{1}(C) \rightarrow \pi_{1}(G)$ is injective and hence, by Theorem (7.3), we see that $G_{2}\left(B_{G}\right)$ contains a free abelian group of rank $n$.

We use Theorem (7.3) to compute $G_{1}\left(B O_{n}\right)$. Note that the center $C$ of $O(n)$ consists of $I$ and $-I$, where $I$ is the identity matrix. The inclusion $C \rightarrow^{i} O(n)$ sends $-I$ into the identity component if $n$ is even and into the component of $O(n)$ corresponding to determinant -1 if $n$ is odd. Thus we have

Corollary (7.6). $G_{1}\left(B O_{n}\right)=\pi_{1}\left(B O_{n}\right) \cong Z_{2}$ if $n$ is odd.

In the case of the unitary group $U(n)$, the center $C$ is $S^{1}$. Since $i_{*}: \pi_{1}(C) \rightarrow$ $\pi_{1}(U(n))$ is the homomorphism from $Z$ to $Z$ given by sending 1 to $n$, we see that $G_{2}\left(B U_{n}\right) \supset n Z$.

Remark (7.7). Results about the evaluation subgroups of $B_{G}$ shed some light on the homology of $B_{G}$ by means of Corollary (1) of [9]: Let $h_{p}: \pi_{i}(X) \rightarrow$ $H_{i}\left(X ; Z_{p}\right)$ be the Hurewicz homomorphism tensored with $Z_{p}$, where $p$ is prime. Suppose $h_{p}(\alpha) \neq 0$ where $\alpha \in G_{i}(X)$. Then $H_{*}\left(X ; Z_{p}\right) \cong A \otimes B$ where $A$ is a graded $Z_{p}$-module with (a) one generator in dimensions $0, i, 2 i, \ldots,(p-1) i$ if $i$ is even, or ( $\mathrm{b})$ one generator in dimensions 0 and $i$ if $i$ is odd. This fact is still true if we let $p=\infty$ and $Z_{\infty}$ be the rationals.

8. Characteristic classes and actions on manifolds. Let $G$ be a group of diffeomorphisms acting on the differentiable manifold $M$. Let $\hat{\omega}: G \times M \rightarrow M$ denote the action. If $k \in H^{*}(M ; \pi)$ is a characteristic class of $M$ and $\pi$ is any group of coefficients, we shall show that $\hat{\omega}^{*}(k)=1 \times k$. Then, using characteristic classes defined by Fadell for the topological case, we shall prove $\hat{\omega}^{*}(k)=1 \times k$ where the $G$ in $\hat{\omega}: G \times M \rightarrow M$ is a group of homeomorphisms.

The above results yield various corollaries, the best of which is: Let $\omega: G \rightarrow$ $M$ be the evaluation map. Then $\chi(M) \omega^{*}: H^{i}(M ; R) \rightarrow H^{i}(G ; R)$ is the zero homomorphism where $i>0, G$ is a group of homeomorphisms on a closed manifold $M$ and $R$ is any coefficient ring and $\chi(M)$ is the Euler-Poincaré number of $M$.

During the course of the proof, we also show that $\tau_{\#}: G \rightarrow B O_{n}^{M}$ is essentially contractible where $\tau_{\#}$ is given by $\tau_{\#}(g)=\tau \circ g$ and $\tau: M \rightarrow B O_{n}$ is the classifying map for the tangent bundle. 
Let $G$ be a space of maps from $M \rightarrow M$ where $M$ is a $C W$ complex, and let $\hat{\omega}: G \times M \rightarrow M$ be the action. Let $\tau(M) \rightarrow M$ be any fibre bundle and let $\tau: M \rightarrow$ $B_{\infty}$ be the classifying map. Now suppose there exists a map $i: G \rightarrow L^{*}(\tau(M), \tau(M))$ such that $j=\Phi \circ i: G \rightarrow L(M, M)$ is induced by the action. Then, we get the following commutative diagram:

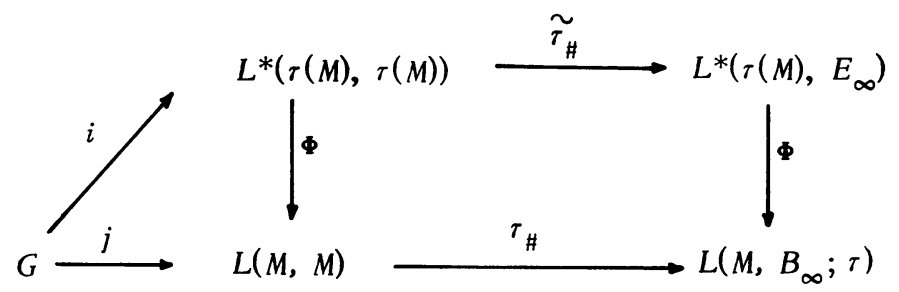

where $j=\Phi \circ i$ and $E_{\infty} \rightarrow B_{\infty}$ is the universal bundle.

Theorem (8.2). With notation above, $\tau_{\#} \circ j$ is essentially bomotopic to a constant map.

Proof. Consider diagram (8.1). Since $L^{*}\left(\tau(M), E_{\infty}\right)$ is essentially contractible, by Theorem 6.2 we see that $\Phi{\widetilde{\tau_{H}}}^{i}$ is essentially homotopic to a constant map and since $\Phi \widetilde{\tau}_{\#} i=\tau_{\#} \circ j$ we have that $\tau_{\#} \circ j$ is essentially homotopic to a constant map.

Remark (8.3). When $G$ is a locally compact $C W$ complex and $r(M)$ is a $k$-space then $\tau_{\#} \circ j$ is actually bomotopic to a constant map.

Corollary (8.4). $\tau \circ \hat{\omega}: G \times M \rightarrow M \rightarrow B_{\infty}$ is essentially bomotopic to $\tau \circ$ (projection).

Proof. The following diagram is commutative.

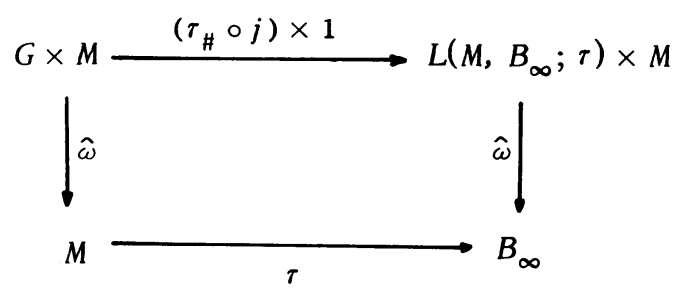

Here the $\hat{\omega}$ on the right is given by $\hat{\omega}(f, x)=f(x)$ for $(f, x) \in L\left(M ; B_{\infty} ; \tau\right) \times M$. Since $\tau_{\sharp} j$ is essentially homotopic to a constant map we have that $\hat{\omega}\left(\tau_{\sharp} j \times 1\right)$ is essentially homotopic to $\tau \circ$ (projection). Since $\tau \circ \hat{\omega}=\hat{\omega} \circ\left(\tau_{\#} j \times 1\right)$, we are done.

Corollary (8.6). Let $k \in H^{*}\left(B_{\infty} ; \pi\right)$. Then

$$
\hat{\omega}^{*}\left(\tau^{*}(k)\right)=1 \times \tau^{*}(k) \in H^{*}(G \times M ; \pi) .
$$

Proof. Since $\tau_{\#} \hat{\omega}$ is essentially homotopic to $\tau \circ$ (projection), we have $1 \times$ $\tau^{*}(k)=(\text { projection })^{*} \circ \tau^{*}(k)=\hat{\omega}^{*}\left(\tau^{*}(k)\right)$. 
Pick a base point $x_{0} \in M$. Then let $\omega: G \rightarrow M$ be the evaluation map given by $\omega(g)=g\left(x_{0}\right)$. Let $R$ be a ring of coefficients and let $u \in H^{i}(M, R)$. Now $\hat{\omega}^{*}(u)=\omega^{*}(u) \times 1$ $+1 \times u+$ other terms where the other terms are represented by chains of the form $\sum a_{i} \times b_{i}$ where the $b_{i}$ have dimension greater than zero. Now we state a fact which will allow us to apply Corollary (8.6) in a very effective manner.

Lemma (8.7). Suppose that $u$ and $v \in \tilde{H}^{*}(M, R)$. Suppose $\hat{\omega}^{*}(v)=1 \times v$. Then $u \cup v=0$ implies $\omega^{*}(u) \times v=0$.

Proof.

$$
\begin{aligned}
0 & =\hat{\omega}^{*}(u \cup v)=\hat{\omega}^{*}(u) \cup \hat{\omega}^{*}(v) \\
& =\left(\omega^{*}(u) \times 1+1 \times u+\text { other terms }\right) \cup 1 \times v \\
& =\omega^{*}(u) \times v+(\text { other terms }) \cup 1 \times v .
\end{aligned}
$$

Since $1 \times v$ can be represented by a cycle $1 \times z$, the other terms are represented by chains of the form $\Sigma a_{i} \times\left(b_{i} \cup z\right)$ and the chains on the right have dimension greater than the dimension of $v$. Thus $\omega^{*}(u) \times v$ cannot cancel out with the other terms of $\hat{\omega}^{*}(u \cup v)$, hence $\hat{\omega}^{*}(u) \times v=0$.

The hypotheses of Theorem (8.2) are satisfied when $G$ is a group of diffeomorphisms acting on a differentiable manifold $M^{n}$ with action $\hat{\omega}: G \times M^{n} \rightarrow M^{n}$. The fibre bundle $\tau(M) \rightarrow M^{n}$ is the tangent vector bundle and the universal fibration is the universal bundle $\gamma^{n} \rightarrow B O_{n}$ (or $\gamma^{n} \rightarrow B S O_{n}$ if $M$ is orientable). Then Theorem (8.2) says that $\tau_{\#}: G \rightarrow L\left(M, B O_{n}\right)$ is essentially homotopic to a constant map. (Here and below we shall write $\tau_{\#}$ for $\tau_{\#} \circ j$, thus suppressing the technical $j$.)

Let us call any $k \in H^{i}(M ; \pi)$ a characteristic class if $k=\tau^{*}(u)$ for some $u \in$ $H^{i}\left(B O_{n}, \pi\right)$ (or $u \in H^{i}\left(B S O_{n}, \pi\right)$ ). Then if $k$ is a characteristic class of $M$, Corollary (8.6) says that $\hat{\omega}^{*}(k)=1 \times k$. This simple relationship apparently was unknown until now, so we will note it as a theorem.

Theorem (8.8). If $k$ is a characteristic class of a differentiable manifold $M$ with $G$ operating as a group of diffeomorphisms with the action $\hat{\omega}: G \times M \rightarrow M$, then $\hat{\omega}^{*}(k)=1 \times k$.

In view of Lemma (8.7), we obtain many results about $\omega^{*}$. The higher the dimension of a characteristic class, the more information we obtain about $\omega^{*}$. It is very desirable to find characteristic classes in the top dimension of $M^{n}$. The Euler class $\chi^{(M) \mu}$ and the index $I(M) \mu$ times some integer $c$ are two such for closed oriented $M$. Here $\mu$ is the fundamental class in the cohomology of the manifold.

Theorem (8.9). Let $M^{n}$ be a closed oriented differentiable manifold and $G$ be a group of diffeomorphisms of $M^{n}$. Then $\chi(M) \omega^{*}: \tilde{H}^{*}(M ; R) \rightarrow \widetilde{H}^{*}(G ; R)$ and 
$c \cdot I(M) \omega^{*}: \widetilde{H}^{*}(M ; R) \rightarrow \widetilde{H}^{*}(G ; R)$ are the zero bomomorphisms for any unitary ring $R$ of coefficients, where $c$ is an integer depending only on the dimension of $M$.

Proof. We shall prove it for $c \cdot I(M)$; the proof for $\chi(M)$ is similar. $I(M) \mu$ is a rational linear combination of Pontrjagin numbers, so $c \cdot I(M) \mu$ is an integral linear combination of products of Pontrjagin classes. We denote by $c \cdot I(M) \mu$ also the image of $c \cdot I(M) \mu$ in $H^{n}(M, R)$ for any coefficient ring $R$. Thus $c \cdot I(M) \mu$ is a characteristic class in $H^{n}(M, R)$ and so $\hat{\omega}^{*}(c \cdot I(M) \mu)=c \cdot I(M) \cdot 1 \times \mu$. Since $\mu \cup \alpha=0$ for any $\alpha \in \tilde{H}^{*}(M, R)$, we have by Lemma (8.7) that $\omega^{*}(\alpha) \times c \cdot I(M) \mu=0$. Thus $c \cdot I(M) \omega^{*}(\alpha) \times \mu=0$ which implies that $c \cdot I(M) \omega^{*}(\alpha)=0$. Since this holds for arbitrary $\alpha$, we have proved the theorem.

Another situation where Theorem (8.2) applies is as follows: Let $M^{n}$ be a topological manifold and let $G$ be a group of homeomorphism acting on $M$ with action $\hat{\omega}: G \times M \rightarrow M$. Let $\tau(M)=M \times M-\Delta$, the deleted product where $\Delta$ is the diagonal. Then $r(M) \rightarrow^{p} M$ will be the fibre bundle with fibre $M-*$ and projection $p\left(x_{1}, x_{2}\right)=x_{1}$. The group of this fibration is the group of homeomorphisms of $M-*$ which is isomorphic to the group $H$ of homeomorphisms of $M$ leaving * fixed.

Now observe that any homeomorphism $f: M \rightarrow M$ can be lifted to a bundle map $\tilde{f}=f \times f: M \times M-\Delta \rightarrow M \times M-\Delta$. Thus there is the required map $i: G \rightarrow$ $L^{*}(\tau(M), \tau(M))$.

Finally, the classifying bundle is $M-* \rightarrow E_{H} \times_{H}(M-*) \rightarrow B_{H}$. Let $\tau: M \rightarrow$ $B_{H}$ be the classifying map. Now Theorem (8.2) says that $\tau_{\#}: G \rightarrow L\left(M, B_{H} ; \tau\right)$ is essentially homotopic to a constant map.

Fadell [6] defines topological characteristic classes as follows. Suppose $F$ $\rightarrow E \rightarrow^{p} B$ is a Hurewicz fibration and suppose $F_{0} \rightarrow E_{0} \rightarrow^{p}{ }^{p} B$ is a fibre subspace in the sense of Fadell [6]. Then $F_{0} \subset F, E_{0} \subset E$ and $\left(E, E_{0}, p, B ; F, F_{0}\right)$ is called a fibred pair.

Theorem (8.10) (Fadell). Let $\left(E, E_{0}, p, B ; F, F_{0}\right)$ be a fibred pair such that

(1) $B$ is arcwise connected,

(2) $\pi_{1}(B, b)$ acts trivially on $H^{*}\left(F, F_{0} ; R\right) \cong H^{*}\left(p^{-1}(b), p^{-1}(b) ; R\right)$,

(3) $H^{*}\left(F, F_{0} ; R\right) \cong H^{*}\left(E^{n}, E^{n}-0 ; R\right)$ where $E^{n}$ is $n$-dimensional Euclidean space.

Then there exist natural "Thom" isomorphisms

$$
\phi: H^{q}(B ; R) \cong H^{n+q}\left(E, E_{0} ; R\right) \text {. }
$$

We say any fibred pair is $R$-orientable if condition (2) of the above theorem holds. Then using $\phi$, Fadell defines natural characteristic classes for $R$-orientable fibred pairs satisfying (3) above. For example, let $U=\phi(1) \in H^{n}\left(E, E_{0} ; R\right)$. Then the Euler class is $\phi^{-1}(U \cup U)$.

Now let $M$ be a connected topological $n$-manifold. Then Fadell shows that the pair $\mathcal{F}=(M \times M, M \times M-\Delta, p, M)$ is a locally trivial fibred pair with fibre $(M, M-*)$ 
where $p$ is projection on the first factor. If $M$ is $R$-orientable in the usual sense, then $\mathcal{F}$ is orientable as a fibred pair. The characteristic classes of this fibred pair will be called the characteristic classes of M. Now R. F. Brown proved the following theorem in [3].

Theorem (8.11) (R. F. Brown). With M a closed oriented connected topological manifold, the Euler class of $M$ is just $\chi(M) \mu$ where $\mu$ is a generator of $H^{n}(M, R)$.

Now we are in a position to prove the analogue for topological manifolds of Theorem (8.8).

Theorem (8.12). Let $\hat{\omega}: G \times M \rightarrow M$ be the action of a connected group $G$ of bomeomorphisms on a closed, R-oriented topological manifold $M$. Let $k \in H^{*}(M, R)$ be a Fadell characteristic class. Then $\hat{\omega}^{*}(k)=1 \times k$.

Proof. Recall that $H$ is the group of homeomorphisms of $M$ which leave the base point $*$ fixed. Let $E_{H} \rightarrow^{p} B_{H}$ be the universal principal fibre bundle with fibre $H$. Let $E=E_{H} \times_{H} M$ and $E_{0}=E_{H} \times_{H}(M-*)$. Then $\left(E, E_{0}, p, M\right)$ is a fibred pair with fibre $(M, M-*)$. As was stated above, the map $\tau: M \rightarrow B_{H}$ classifies the bundle $M \times M-\Delta \rightarrow M$. Thus $\tau_{\#}$ is essentially homotopic to a constant.

Now we want to know that $k \in H^{*}(M, R)$ is equal to an element $\bar{k} \in H^{*}\left(B_{H}, R\right)$ pulled back by $\tau^{*}$, that is, $k=\tau^{*}(\bar{k})$. Since the definition of characteristic classes is natural, we only need to define the appropriate characteristic class for the fibred pair $\left(E, E_{0}\right)$ and we are done. Unfortunately, except for when $R=Z_{2}$, the fibred pair $\left(E, E_{0}\right)$ need not be orientable. So we consider the covering space $\widetilde{B}_{H}$ corresponding to the subgroup $S \subset \pi_{1}\left(B_{H}\right)$ of all elements operating trivially on $H^{n}(M, M-*)$. If $p: \widetilde{B}_{H} \rightarrow B_{H}$ is the covering projection, the fibred pair $\left(E, E_{0}\right)$ pulls back to a fibred pair $\left(p^{*} E, p^{*} E_{0}\right)$ over $\widetilde{B}_{H}$. Now $\left(p^{*} E, p^{*} E_{0}\right)$ is orientable, hence we can define a characteristic class $\bar{k} \in H^{*}\left(\widetilde{B}_{H}, R\right)$ which pulls back to $k$ under any map $\bar{\tau}: M \rightarrow \widetilde{B}_{H}$ which is induced by the fibre bundle map of the two appropriate fibre pairs.

Since $(M \times M, M \times M-\Delta)$ is orientable, the image of $\tau_{*}: \pi_{1}(M) \rightarrow \pi_{1}\left(B_{H_{2}}\right)$ is contained in $S$. Thus $\tau$ factors through $\widetilde{B}_{H}$; that is, there exists a $\bar{\tau}: M \rightarrow \widetilde{B}_{H}$ such that $\tau=p \circ \bar{\tau}$. This $\bar{\tau}$ is induced by fibre bundle maps of fibre pairs, so $\bar{\tau} *(\bar{k})=k$.

Now it only remains to show that $\overline{\tau_{\#}}$ is essentially homotopic to a constant. Note that $L\left(M, \widetilde{B}_{H} ; \bar{\tau}\right) \rightarrow^{p_{\#}} L\left(M, B_{H} ; \tau\right)$ is a fibration with discrete fibre. Since $\tau_{\#}: G \rightarrow L\left(M, B_{H} ; \tau\right)$ is essentially homotopic to a constant map, $\tau_{\#}$ restricted to any compact set $X$ of $G$ is homotopic to a constant. By the covering homotopy property, the homotopy lifts to a homotopy in $L\left(M, \widetilde{B}_{H} ; \bar{\tau}\right)$ and at the last stage results in a map from $X$ into the discrete fibre. Hence $\bar{\tau}_{\#} \mid X$ is homotopic to a constant. Hence $\bar{\tau}_{\#}$ is essentially homotopic to a constant.

Using the appropriate version of diagram (8.5) we see that $\bar{\tau} \circ \hat{\omega}$ is essentially 
homotopic to $\tau \circ$ (projection). Hence $\hat{\omega}^{*}(k)=\hat{\omega}^{*} \circ \bar{\tau}^{*}(\bar{k})=($ proj. $) *(k)=1 \times k$, thus proving the theorem.

Theorem (8.13). Let $M$ be a closed connected oriented topological manifold

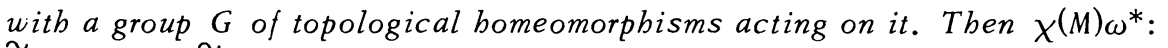
$\tilde{H}^{*}(M, R) \rightarrow \tilde{H}^{*}(G, R)$ is trivial.

Proof. Use Brown's Theorem (8.11) to show that $\hat{\omega}^{*}(\chi(M) \mu)=1 \times \chi(M) \mu$. Then Lemma (8.7) as before gives the desired result.

Remark (8.14). In the above theorems, if $R=Z_{2}$ we do not need $M$ to be oriented.

Remark (8.15). (a) If $k$ is a characteristic class, then $\hat{\omega}^{*}(k)=1 \times k$ implies $\omega^{*}(k)=0$. Since $\omega^{*}$ is injective if $M$ is a topological group, this shows the well known fact that characteristic classes of topological groups are zero.

(b) $\tau_{\#}$ essentially homotopic to a constant shows that $\tau \circ \omega$ is essentially homotopic to a constant. This yields another proof to the statement that the tangent bundle of an odd sphere is of order two. In fact, we have Diff $S^{2 n+1} \rightarrow^{\omega}$ $S^{2 n+1} \rightarrow{ }^{\tau} \mathrm{BSO}_{n}$ and the image of $\omega_{*}: \pi_{2 n+1}\left(\right.$ Diff $\left.S^{2 n+1}\right) \rightarrow \pi_{2 n+1}\left(S^{2 n+1}\right) \cong Z$ is the even integers except for $S^{1}, S^{3}$ and $S^{7}$. If $\alpha \in \pi_{2 n+1}\left(S^{2 n+1}\right)$ is a generator, we have $\tau_{*}(2 \alpha)=\tau_{*}\left(\omega_{*}(\bar{\alpha})\right)=0$.

(c) Let $G$ be a topological group and let $H$ be a closed subgroup. Then if $G / H$ is a closed oriented manifold, then $\chi(G / H) \rho^{*}$ is trivial where $\rho: G \rightarrow G / H$ is the coset map. This follows from the above theorem since $\rho$ may be regarded as $\omega$.

(d) The Euler-Poincaré number in Theorem (8.13) is essential. For example, $\rho: \mathrm{SO}_{3} \rightarrow \mathrm{SO}_{3} / \mathrm{SO}_{2}=\mathrm{S}^{2}$ induces the homomorphism $\rho^{*}: H^{2}\left(S^{2}, Z_{2}\right) \rightarrow H^{2}\left(\mathrm{SO}_{3}, Z_{2}\right)$ which is not the zero homomorphism.

Added in proof. Theorem (8.13) can be generalized to hold for any compact manifold with or without boundary at a slight cost to the conclusion $\left(4 \chi(M) \omega^{*}=0\right)$.

9. Vector bundle monomorphisms. The theory of immersions of a manifold into another manifold gives rise to the space of immersions $\operatorname{Im}(M, W)$ where $M$ and $W$ are differential manifolds without boundary. Any immersion $f$ induces a map on the tangent bundles $\tilde{f}: \tau(M) \rightarrow \tau(W)$. $\tilde{f}$ maps fibres into fibres, and $\tilde{f}$ restricted to any fibre is an injective linear map of an $n$-dimension Euclidean vector space into an $m$-dimensional Euclidean vector space. We call such a map (i.e. fibre preserving and restricts to fibres as an injective linear transformation) a vector bundle injection.

Let $\xi^{n}$ be an $n$-dimensional vector bundle and let $\eta^{m}$ be an $m$-dimensional one. Then let $L^{*}\left(\xi^{n}, \eta^{m}\right)$ be the space of vector bundle injections endowed with the compact-open topology. Then the space of immersions has the same weak homotopy type as $L^{*}(\tau(M), \tau(W))$ if $\operatorname{dim} M<\operatorname{dim} W$ or if $M$ is open and $\operatorname{dim} M=\operatorname{dim} W$. See [13, Theorem 8.4], for example.

Thus, it is interesting to study the space of vector bundle injections for any 
pair of vector bundles, $\xi^{n}$ and $\eta^{m}$. So suppose we have the vector bundle injection

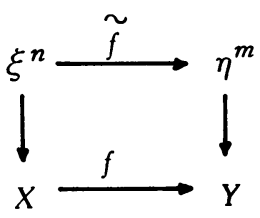

Now $f^{*}\left(\eta^{m}\right)$ is a vector bundle over $X$ and so $f^{*}\left(\eta^{m}\right)=\xi^{n} \oplus \nu^{m-n}$ where $\oplus$ denotes Whitney sums and $\nu^{m-n}$ is a vector bundle "orthogonal" to $\xi^{n}$ in $f^{*}\left(\eta^{m}\right)$. Now consider $L^{*}(\xi \oplus \nu, \eta)$, the space of vector bundle maps. The restriction of any vector bundle map to $\xi$ in $\xi \oplus \nu$ is a vector bundle injection. We shall let $\Phi: L^{*}(\xi \oplus \nu, \eta) \rightarrow L^{*}(\xi, \eta)$ be this restriction. Then $\Phi$ is a Serre fibration, see A. Phillips [13, proof of Theorem 8.4].

Let $\hat{L}^{* *}(\xi \oplus \nu, \eta)$ be the space of vector bundle maps inducing $\tilde{f}: \xi \rightarrow \eta$. Then $\hat{L}^{* *}(\xi \oplus \nu, \eta)$ is the fibre of the fibration $\Phi$. Now $\hat{L}^{* *}(\xi \oplus \nu, \eta)$ is homeomorphic to $\hat{L}^{* *}(\xi \oplus \nu, \xi \oplus \nu)$, the space of bundle maps whose restriction to $\xi \subset \xi \oplus \nu$ is the inclusion $\xi \rightarrow \xi \oplus \nu$.

Consider the projections $p: \xi \oplus \nu \rightarrow \nu$ and $q: \xi \oplus \nu \rightarrow \xi$ and the injection $i: \nu \rightarrow \xi \oplus \nu$. Now there is a homomorphism $b: L^{* *}(\nu, \nu) \rightarrow \hat{L}^{* *}(\xi \oplus \nu, \xi \oplus \nu)$ given by $b(\tilde{f})=1 \oplus \tilde{f}$. Recall from $\S 4$ that $L^{* *}(\nu, \nu)$ denotes the space of self bundle equivalences. Note $b$ is a homomorphism with composition of functions as the multiplication on $L^{* *}$ and $\hat{L}^{* *}$.

Theorem (9.1). $b$ is a bomotopy equivalence.

Proof. Let $p: \xi \oplus \nu \rightarrow \nu$ and $q: \xi \oplus \nu \rightarrow \xi$ be projections and let $i: \nu \rightarrow$ $\xi \oplus \nu$ be the inclusion. Define a map $k: \hat{L}^{* *}(\xi \oplus \nu, \xi \oplus \nu) \rightarrow L^{* *}(\nu, \nu)$ by $k(\widetilde{g})$ $=p \circ \widetilde{g} \circ i$. Then $k \circ b=$ identity on $L^{* *}(\nu, \nu)$.

We shall prove that $b \circ k$ is homotopic to the identity on $\hat{L}^{* *}$. Thus $k$ is a homotopy inverse of $b$ which is what we need to prove.

Let $\widetilde{g} \in \hat{L}^{* *}(\xi \oplus \nu, \xi \oplus \nu)$. Then $b \circ k(\widetilde{g})=1 \oplus p \widetilde{g} i$. Thus we need to find a systematic manner to homotopy $\tilde{g}$ into $1 \oplus p \tilde{g} i$. Since $\tilde{g} \mid \xi$ is the identity on $\xi$ for all $\widetilde{g} \in \hat{L}^{* *}$, we see that $\widetilde{g}$ is uniquely determined by $\widetilde{g} \circ i: \nu \rightarrow \xi \oplus \nu$. Thus we wish to find a systematic homotopy from $\tilde{g} \circ i$ to $p \tilde{g} i$.

Now $\tilde{g} \circ i$ is determined by $p \circ \tilde{g} \circ i$ and $q \circ \tilde{g} \circ i$, the first of which is a vector bundle injection and the second is only a vector bundle morphism. Thus, $\tilde{g} \circ i=q \circ \tilde{g} \circ i+p \circ \tilde{g} \circ i$ where + means vector sum in each fibre. Define a homotopy of vector bundle injections by $\tilde{g}_{t}=t \cdot q \circ \tilde{g} \circ i+p \circ \tilde{g} \circ i$. Then $\tilde{g}_{0}=p \circ$ $\widetilde{g} \circ i$ and $\widetilde{g}_{1}=q \circ \widetilde{g} \circ i+p \circ \widetilde{g} \circ i=\widetilde{g}$.

Now let us consider the universal $n$-vector bundle $\gamma^{n} \rightarrow G_{n}$ where $G_{n}$ is the Grassmannian. Now we have two fibrations: 


$$
\hat{L}^{* *}(\xi \oplus \nu, \xi \oplus \nu) \rightarrow L^{*}\left(\xi \oplus \nu, \gamma^{m}\right) \stackrel{\Phi}{\rightarrow} L^{*}\left(\xi^{n}, \gamma^{m}\right)
$$

and

$$
L^{* *}(\nu, \nu) \rightarrow L^{*}\left(\nu, \gamma^{m-n}\right) \stackrel{\Phi}{\rightarrow} L\left(X, G_{m-n} ; \nu\right) .
$$

Now both $L^{*}\left(\xi \oplus \nu, \gamma^{m}\right)$ and $L^{*}\left(\nu, \gamma^{m-n}\right)$ are essentially contractible, hence weakly contractible (all homotopy groups are trivial). Since $L^{* *}$ and $\hat{L}^{* *}$ are both $H$-spaces and $b: L^{* *} \rightarrow \hat{L}^{* *}$ is an $H$-mapping which is a homotopy equivalence, we are led to suspect the following fact:

Theorem (9.2). $L^{*}\left(\xi^{n}, \gamma^{m}\right)$ is weak bomotopy equivalent to $L\left(X, G_{m-n} ; \nu\right)$.

Remark (9.3). Notice that if $X$ is a point, then $\xi^{n}$ is just $R^{n}, n$-dimensional Euclidean space. Then Theorem (9.2) says that $L^{*}\left(R^{n}, \gamma^{m}\right)$ is homotopy equivalent to $G_{m-n}$ as is well known.

Proof of Theorem. The predicted weak homotopy equivalence $f: L^{*}\left(\xi, \gamma^{m}\right) \rightarrow$ $L\left(X, G_{m-n} ; \nu\right)$ can be realized as follows: We consider $G_{m}$ as the space of $m$ dimensional subspaces in $R^{\infty}$, the direct limit of the $R^{n}$ 's. Let $\widetilde{g} \in L^{*}\left(\xi^{n}, \gamma^{m}\right)$ and let $x \in X$. We define $f(\tilde{g})(x) \in G_{m-n}$ to be the $(m-n)$-dimensional subspace of $R^{\infty}$ as follows. The fibre over $x$ in $\xi^{n}$ is $R^{n}$, and its image under $\tilde{g}$ is an $n$ dimensional subspace, $S_{\xi}$, of the $m$-dimensional subspace, $S_{x}$, given by $g(x) \in G_{m}$. Let $S_{\xi}^{1}$ be the $(m-n)$-dimensional subspace of $S_{x}$ orthogonal to $S_{\xi}$. Then $S_{\xi}^{\frac{1}{m}}$ represents a point in $G_{m-n}$ which we denote by $f(\tilde{g})(x)$.

To see that $f$ is a homotopy equivalence, we define $\tilde{f}: L^{*}\left(\xi \oplus \nu, \gamma^{m}\right) \rightarrow$ $L^{*}\left(\nu, \gamma^{m-n}\right)$ as follows: Let $\tilde{g} \in L^{*}\left(\xi \oplus \nu, \gamma^{m}\right)$ and let $v \in \nu$ be a vector in $\nu$. Then define $\tilde{f}(\widetilde{g})(v)=p \tilde{g}(v)$ where $p$ denotes the projection from $S_{x} \rightarrow S_{\xi}^{1}$ (where $v$ is in the fibre of $x$ ). Then $\tilde{f}$ is a fibre preserving map and we have the commutative diagram

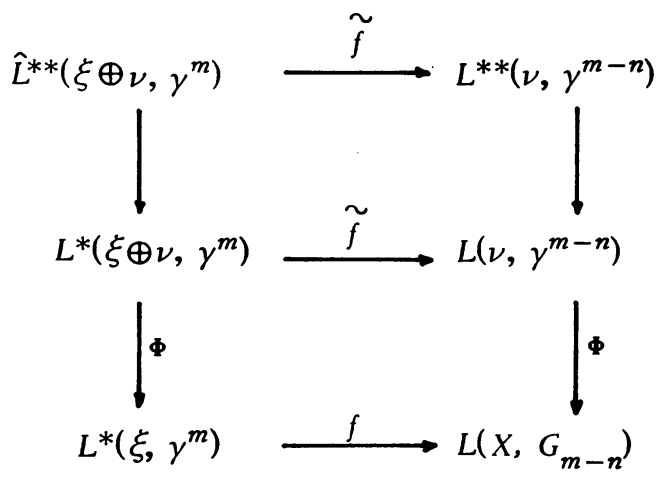

A little reflection shows that the top $\tilde{f}$ is the same as $k$ in the proof of Theorem (9.1), and hence is a homotopy equivalence. Since both middle $L^{*}$ 's are essentially contractible, we see that $f$ is a weak homotopy equivalence. 


\section{PART III. THE GNAW EXACT SEQUENCE}

In this part we apply bundle map theory to get an exact sequence which is "dual" to the cohomology Wang exact sequence. Then we apply the exact sequence to $H$-spaces and to evaluation subgroups.

10. The Wang homomorphism. For a particular $a \in \pi_{n}\left(F^{F}\right)$, we have a Hurewicz fibration $F \rightarrow^{i} E \rightarrow^{p} a S^{n+1}$ and so we have the Wang exact sequence corresponding to $\alpha$. Namely,

$$
\cdots \rightarrow H^{q}(E ; G) \stackrel{i^{*}}{\rightarrow} H^{q}(F ; G) \stackrel{\lambda_{\alpha}}{\rightarrow} H^{q-n}(F ; G) \rightarrow H^{q+1}(E, G) .
$$

We shall call $\lambda_{a}$ the Wang bomomorpbism for $\alpha$.

There is another definition of $\lambda_{\alpha}$. Suppose $\alpha: S^{n} \rightarrow F^{F}$ denotes a map which represents $\alpha \in \pi_{n}\left(F^{F}\right)$. Then we have the commutative diagram which defines $\hat{\alpha}$ :

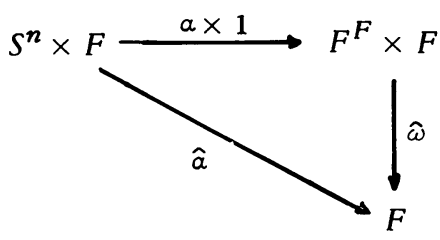

Then, by p. 456 of [15], we have

$$
\hat{\alpha}^{*}(k)=1 \times k+\bar{\sigma} \times \lambda_{\alpha}(k)
$$

where $\bar{\sigma} \in H^{n}\left(S^{n} ; Z\right)$ is a generator.

Let $\sigma \in H_{n}\left(S^{n} ; Z\right)$ be the dual generator to $\bar{\sigma}$, let $b$ be the Hurewicz homomorphism. Using the definition of the slant product on p. 351 of [15], we have

$$
\lambda_{\alpha}(k)=\sigma \backslash \hat{\alpha}^{*}(k)=\sigma \backslash(\alpha \times 1)^{*} \hat{\omega}^{*}(k)=\alpha_{*}(\sigma) \backslash \hat{\omega}^{*}(k)=b(\alpha) \backslash \hat{\omega}^{*}(k) .
$$

This proof is in the spirit of [14].

This fact has two immediate corollaries. The first is: If $b(\alpha)=0$, then $\lambda_{a}$ is the zero homomorphism. The second corollary is:

$$
\lambda_{a+\beta}=\lambda_{a}+\lambda_{\beta}
$$

In view of (10.4), we have a bilinear map from $\pi_{*}\left(F^{F}\right) \times H^{*}(F ; G) \rightarrow H^{*}(F ; G)$ given by $(\alpha, k) \rightarrow \lambda_{\alpha}(k)$. If we fix $\alpha$, we get the Wang homomorphism. If we fix $k$, we get a homomorphism $\Lambda_{k}: \pi_{*}\left(F^{F}\right) \rightarrow H^{*}(F ; G)$ given by $\Lambda_{k}(\alpha)=\lambda_{\alpha}(k)$. We shall call $\Lambda_{k}$ the Gnaw bomomorphism. In the next section, we shall imbed $\Lambda_{k}$ in the Gnaw exact sequence.

Finally, we should note one other important property of $\lambda_{a}$. Let $u \in H^{p}(F ; G)$ and $v \in H^{q}(F ; G)$ and $\alpha \in \pi_{n}\left(F^{F}\right)$. Then 


$$
\lambda_{\alpha}(u \cup v)=\lambda_{\alpha}(u) \cup v+(-1)^{n p} u \cup \lambda_{a}(v) .
$$

11. The Gnaw exact sequence. We wish to study the Gnaw homomorphism $\Lambda_{k}$ where $k \in H^{n}(X ; \pi)$ and $X$ is a CW complex. We shall apply the results of Part I to obtain an exact sequence involving $\Lambda_{k}$.

Dold and Thom [4] and also Milnor have shown that Eilenberg-Mac Lane spaces $K(\pi, n)$ may be regarded as abelian topological groups if $\pi$ is abelian. Thus we have the universal principal fibre bundle $E_{G} \rightarrow K(\pi, n)$ with fibre $G=K(\pi, n-1)$.

We may regard $k \in H^{n}(X, \pi)$ as a map, also denoted $k$, such that $k: X \rightarrow$ $K(\pi, n)$. Then $k$ induces a fibration $E \rightarrow X$ such that

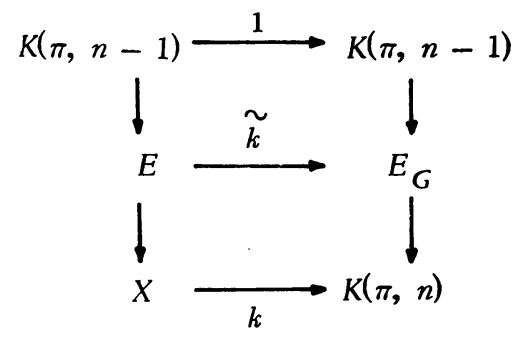

commutes. This leads to the following diagram:

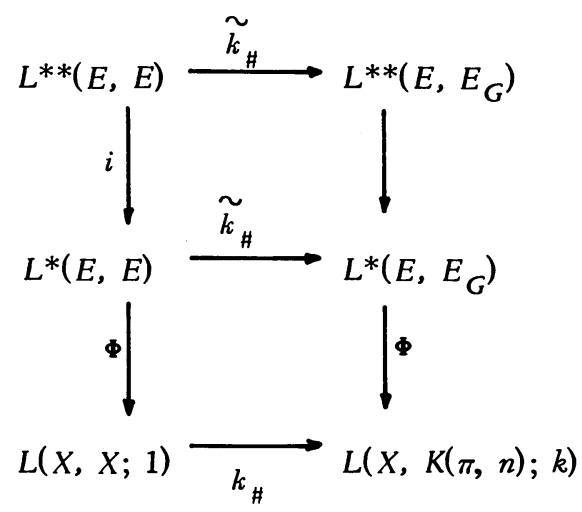

Here $k_{\#}$ is the map given by $k_{\#}(f)=k \circ f$, similarly for $\tilde{k}_{\#}$.

Lemma (11.2). $\pi_{i}(L(X, K(\pi, n) ; k)) \cong H^{n-i}(X ; \pi)$ for $i>0$.

Proof. This is a theorem of Thom [19, Théorème 2]. We shall give the proof here since it is instructive. A map $f: S^{i} \rightarrow L(X, K(\pi, n) ; k)$ corresponds to a map $F: S^{i} \times X \rightarrow K(\pi, n)$ which restricts on $* \times X$ to $k$. If $\iota$ is the fundamental class in $H^{n}(K(\pi, n) ; \pi)$, then

$$
F^{*}(\iota)=1 \times k+\sigma^{i} \times \mu_{n-i} \text { in } H^{n}\left(S^{i} \times X ; \pi\right)
$$


where $\sigma^{i}$ is a generator of $H^{i}\left(S^{i}\right)$. Thus we see that the homotopy classes of $F$ and elements $\mu_{n-i}$ in $H^{n-i}(X ; \pi)$ are in one-to-one correspondence.

Lemma (11.3). The bomomorphism

$$
\left(k_{\#}\right)_{*}: \pi_{i}(L(X, X ; 1)) \rightarrow \pi_{i}(L(X, K(\pi, n) ; k)) \cong H^{n-i}(X ; \pi)
$$

is equal to $\Lambda_{k}: \pi_{i}(L(X, X ; 1)) \rightarrow H^{n-i}(X ; \pi)$ for all $i>0$.

Proof. If $\alpha \in \pi_{i}(L(X, X ; 1))$, we have $\left(k_{\#}\right)_{*}(\alpha)$ represented by a composition $S^{i} \times X \rightarrow^{F} X \rightarrow^{k} K(\pi, n)$ where $F$ represents $\alpha$. Now,

$$
(k \circ F)^{*}(\iota)=F^{*}(k)=1 \times k+\sigma^{i} \times \lambda_{\alpha}(k) .
$$

Thus $\alpha$ is mapped to $\lambda_{\alpha}(k)=\Lambda_{k}(\alpha)$.

Theorem (11.4). There is a long exact sequence $\cdots \rightarrow \pi_{i}\left(X^{X}\right) \rightarrow{ }^{\Lambda_{k}} H^{n-i}(X ; \pi)$ $\rightarrow \pi_{i-1}\left(L^{*}(E, E)\right) \rightarrow^{\Phi^{*}} \pi_{i-1}\left(X^{X}\right) \rightarrow^{\Lambda_{k}} \ldots$.

Proof. From diagram (11.1) and the lemmas above, we get the exact ladder

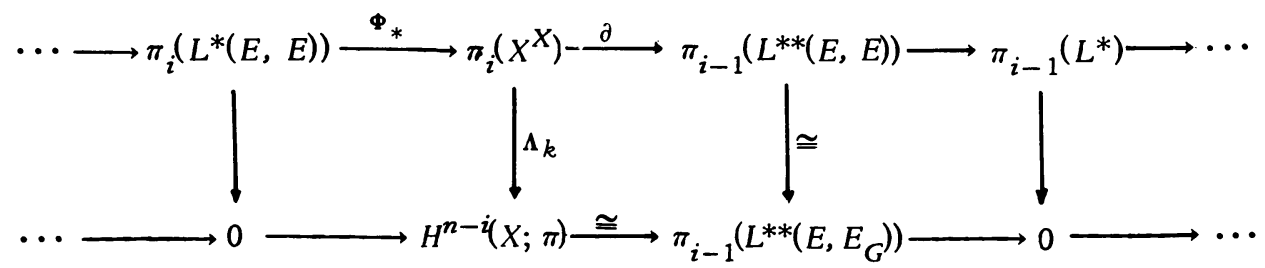

Then, in the top sequence, we replace $\pi_{i-1}\left(L^{* *}\right)$ by $H^{n-i}(X ; \pi)$.

We shall call the above exact sequence the Gnaw exact sequence.

12. Exact sequences of Thom and the Gnaw exact sequence. In this section we shall recall an exact sequence occurring in Thom's paper [19]. When $X$ is an $H$-space, this sequence and the Gnaw exact sequence look comparable. We investigate the relationship between them and show that mostly they are quite different, but in certain circumstances they are very similar. As a result of our investigation, we find an unexpected relation among Gnaw homomorphisms of $H$-spaces.

Thom, in [19], has made use of the fibration

$$
L(A, F) \rightarrow L(A, E ; k) \rightarrow L(A, B ; *)
$$

arising from the fibration $F \rightarrow E \rightarrow^{p} B$. Here * stands for the constant map and $p \circ k \sim *$. When $B$ is an $H$-group, then $L\left(A, B_{;}^{*}\right)$ is homotopically equivalent to $L(A, B ; f)$ for any $f: A \rightarrow B$. Let us suppose that $F \rightarrow E \rightarrow^{p} B$ is the principal fibration, with $F=K(\pi, n-1)$, corresponding to $k \in H^{n}(B ; \pi)$. Then $\pi_{i}(L(B, F))$ $\cong H^{n-i-1}(B ; \pi)$ and we get the exact sequence

$$
\cdots \rightarrow H^{n-i-1}(B ; \pi) \rightarrow \pi_{i}(L(B, E)) \rightarrow \pi_{i}\left(B^{B}, 1\right) \stackrel{o}{\rightarrow} H^{n-i}(B ; \pi) \rightarrow \cdots
$$


Since two out of the three terms agree with the Gnaw exact sequence, it is worthwhile to ask, when do the two sequences agree?

Let $\mu: B \times B \rightarrow B$ be the multiplication on $B$ and let $c: B \rightarrow B$ be the map which is the homotopy inverse, i.e. $\mu(c(x), x) \sim e$ where $e \in B$ is the identity element. Let us denote $\mu(x, y)$ by $x \cdot y$. Now the map $c_{\mu}: L(B, B ; 1) \rightarrow L(B, B ; 0)$ given by $c_{\mu}(f)=\mu(c \times f) \Delta=c \cdot f$ is a homotopy equivalence.

Suppose that $F: S^{i} \times B \rightarrow B$ represents an element $\alpha \in \pi_{i}(L(B, B ; 1))$ and $F^{\prime}: S^{i} \times B \rightarrow B$ represents $\left(c_{\mu_{*}}\right)_{*}(\alpha)$. Then we can choose $F^{\prime}$ such that $F^{\prime}(s, x)=$ $c(x) \cdot F(s, x)$. We may write $F^{\prime}(s, x)$ as the composition of

$$
(s, x) \stackrel{1 \times \Delta}{\longmapsto}(s, x, x) \stackrel{T \times 1}{\longrightarrow}(x, s, x) \stackrel{c \times F}{\longrightarrow}(c(x), F(s, x)) \stackrel{\mu}{\longmapsto} c(x) \cdot F(s, x) .
$$

Define $\lambda_{a}^{\prime}(k)$ by $\left(F^{\prime}\right)^{*}(k)=\sigma \times \lambda_{\alpha}^{\prime}(k)$. We are going to express $\lambda_{\alpha}(k)$ in terms of $\lambda_{a}^{\prime}(k)$ when the coefficients $\pi$ are a field. Suppose that

$$
\mu^{*}(k)=k \& 1+1 \otimes k+\sum k_{j} \otimes k_{j}^{\prime} .
$$

Lemma (12.1). If $\pi$ is a field, with the notation above,

$$
\lambda_{a}^{\prime}(k)=\lambda_{\alpha}(k)+\sum_{j}(-1)^{i \cdot d(j)} c^{*}\left(k_{j}\right) \cup \lambda_{a}\left(k_{j}^{\prime}\right)
$$

where $d(j)$ is the dimension of $k_{j}$.

Proof. We use the composition $\left(F^{\prime}\right)^{*}=(1 \times \Delta)^{*}(T \times 1)^{*}(c \times F)^{*} \mu^{*}$. Thus

$$
\begin{aligned}
k \stackrel{\mu^{*}}{\longrightarrow} & k \otimes 1+1 \otimes k+\sum k_{j} \otimes k_{j}^{\prime} \\
\stackrel{c^{*} \times F^{*}}{\longrightarrow} & c^{*}(k) \otimes 1 \otimes 1+1 \otimes\left(1 \otimes k+\sigma \otimes \lambda_{a}(k)\right) \\
& +\sum_{j} c^{*}\left(k_{j}\right) \otimes\left(1 \otimes k_{j}^{\prime}+\sigma \otimes \lambda_{a}\left(k_{j}^{\prime}\right)\right) \\
& \stackrel{T^{*} \times 1}{\longrightarrow} 1 \otimes c^{*}(k) \otimes 1+1 \otimes 1 \otimes k+\sigma \otimes 1 \otimes \lambda_{a}(k) \\
& +\sum_{j} 1 \otimes c^{*}\left(k_{j}\right) \otimes k_{j}+\sum_{j}(-1)^{i d(j)} \sigma \otimes c^{*}\left(k_{j}\right) \otimes \lambda_{a}\left(k_{j}^{\prime}\right) \\
& \stackrel{1 \times \Delta^{*}}{\longrightarrow} 1 \otimes\left(c^{*}(k)+k+\sum_{j} c^{*}\left(k_{j}\right) \cup k_{j}^{\prime}\right) \\
& +\sigma \otimes\left(\lambda_{a}(k)+\sum_{j}(-1)^{i d(j)} c^{*}\left(k_{j}\right) \cup \lambda_{a}\left(k_{a}^{\prime}\right)\right) .
\end{aligned}
$$

Now $k+c^{*}(k)+\Sigma_{j} c^{*}\left(k_{j}\right) \cup k_{j}^{\prime}=0$ and the term tensored with $\sigma$ must be $\lambda_{a}^{\prime}(k)$.

As a consequence of this lemma we have $\Lambda_{k}^{\prime}=\Lambda_{k}+\Sigma_{j}(-1)^{i d(j)} c^{*}\left(k_{j}\right) \cup \Lambda_{k_{j}}$, where $\Lambda_{k}^{\prime}$ is defined by $\Lambda_{k}^{\prime}(\alpha)=\lambda_{a}^{\prime}(k)$. Now $\Lambda_{k}^{\prime}$ is the homomorphism $\partial^{j}$ in the Thom exact sequence which we are comparing to our Gnaw exact sequence. If $k$ is a primitive element, i.e. $\mu^{*}(k)=1 \otimes k+k \otimes 1$, then $\Lambda_{k}^{\prime}=\Lambda_{k}$ and the two exact 
sequences are very similar. In fact, two out of the three terms of the two sequences agree and the connecting homomorphisms are the same. In the absence of a commuting map from $L(B, E ; *)$ to $L^{*}(E, E)$, we can only use the " $4 \frac{1}{2} / 2$ lemma" to show that $\pi_{i}\left(L^{*}(E, E)\right)$ and $\pi_{i}(L(B, E ; *))$ are in one-to-one correspondence with each other.

Now, as is well known, $\lambda_{\alpha}(u \cup v)=\lambda_{\alpha}(u) \cup v \pm u \cup \lambda_{\alpha}(v)$. On the other hand, $\lambda_{a}^{\prime}(u \cup v)=0$ since $\left(F^{\prime}\right)^{*}(u \cup v)=0 \quad(u$ and $v$ are assumed to have positive dimension). This can be seen since $\left(F^{\prime}\right)^{*}(x)=\sigma \otimes \lambda_{\alpha}^{\prime}(x)$. Thus $\left(F^{\prime}\right)^{*}(u \cup v)=$ $\left(\sigma \otimes \lambda_{\alpha}^{\prime}(u)\right) \cup\left(\sigma \otimes \lambda_{\alpha}^{\prime}(v)\right)=0$ since $\sigma \cup \sigma=0$. Thus if $k$ is decomposable in $H^{*}(B ; \pi)$, then $\Lambda_{k}^{\prime}$ is the trivial map, whereas $\Lambda_{k}$ may not be. So, in general, the Thom exact sequence and the Gnaw exact sequence are quite different.

Lemma (12.1) gives us an interesting relation among the Gnaw homomorphisms.

Theorem (12.2). If $k \in H^{n}(B ; \pi)$ where $\pi$ is a field, $B$ is an $H$-group and $k$ is decomposable, then $\Lambda_{k}=-\Sigma_{j}(-1)^{i d(j)} c^{*}\left(k_{j}\right) \cup \Lambda_{k_{j}}$ where $\Lambda_{k}: \pi_{i}\left(X^{X}\right) \rightarrow$ $H^{n-i}(X ; \pi)$.

13. Some notes on [8]. In this section, we shall point out a shorter proof and improve a result in [8]. Let $K(\pi, n) \rightarrow E \rightarrow^{p} X$ be the principal fibre bundle corresponding to $k \in H^{n+1}(X ; \pi)$. Then Theorem 6.3 of [8] states

Theorem (13.1). There exists a map $\tilde{\varphi}: S^{i} \times E \rightarrow E$ such that $\tilde{\varphi} \mid E$ is the identity on $E$ and

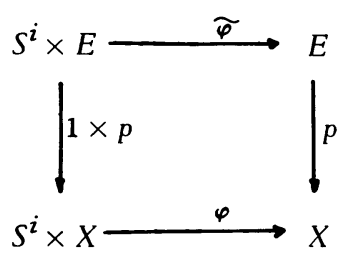

commutes if and only if $\lambda_{\alpha}(k)=0$ where $\alpha$ is represented by $\varphi$.

Proof. From Theorem (11.4), we know that $\pi_{i}\left(L^{*}(E, E)\right) \rightarrow^{\mathbf{\Phi}} \pi_{i}\left(X^{X}\right)$ $\rightarrow{ }^{\Lambda_{k}} H^{n-i}(X ; \pi)$ is exact. Translating this exactness property into associated maps, we essentially get the theorem. In fact, if $\lambda_{\alpha}(k)=0$, then there exists a principal bundle map $\tilde{\varphi}$, as in the theorem, by exactness. If, on the other hand, there exists a $\widetilde{\varphi}$ as in the theorem, the commutativity of the diagram shows that $\lambda_{\alpha}(k)=0$.

This is a useful theorem. It was used many times in [8]. Suppose that we have the sequence of fibrations $\cdots \rightarrow X_{N} \rightarrow^{p}{ }^{N} X_{N-1} \rightarrow \cdots \rightarrow X_{1} \rightarrow^{p}{ }^{1} X$ where $X_{N}$ is the $N$ connective covering of $X$. Then the above theorem easily implies that $\left(p_{N}\right)_{*}\left(G_{i}\left(X_{N}\right)\right) \supset G_{i}\left(X_{N-1}\right)$ for $i>N([8$, Corollary 6.5]). Now we shall state and prove a better version of Theorem 7.2 of [8]. 
Theorem (13.2). Suppose $X$ is a CW complex with a finite number of nonzero rational bomology groups. Let $\pi_{*}(X)$ be of finite type and suppose that $\pi_{i}(X)$ is a finite group for all odd $i, 1<i<2 N$. Then $G_{i}(X)$ is a finite subgroup for all even $i$ for $i \leq 4 N$.

Proof. The conditions that $H_{*}(X ; R)$ is finite dimensional and that odd homotopy groups are finite for dimensions less than $2 N$ allows us to conclude that $H_{*}\left(X_{i} ; R\right)$ is finite dimensional for $i \leq 2 N$ by means of the Serre spectral sequence mod finite groups. Observe that Remark (7.7), when $p=\infty$, tells us that, for $\alpha \epsilon$ $G_{i+1}\left(X_{i}\right)$, we have $b_{\infty}(\alpha)=0$ when $i \leq 2 N$. Since $X_{i}$ is $i$-connected, $b_{\infty}$ is an isomorphism mod finite groups, so $G_{i+1}\left(X_{i}\right)$ is a finite group, hence $G_{i}(X)$ is a finite group for $i \leq 2 N$. Now $X_{2 N}$ is $2 N$-connected, so by a well-known theorem (see [12, p. 108]) we have that the Hurewicz map $b: \pi_{i}\left(X_{2 N}\right) \rightarrow H_{i}\left(X_{2 N}\right)$ is an isomorphism mod finite groups for $i \leq 4 N$. Hence applying Remark (7.7) again, we find that $G_{i}\left(X_{2 N}\right)$ is finite for even $i \leq 4 N$. Hence $G_{2 i}(X)$ is finite for $i \leq 2 N$.

Corollary (13.3). Suppose we have a fibration $F \rightarrow E \rightarrow^{p} B$ and $F$ bas finite dimensional rational bomology and $\pi_{*}(F)$ is of finite type. If $\pi_{i}(F)$ is a finite abelian group for all odd $i$ such that $1<i<2 N$, then $p_{*}: \pi_{i}(E) \rightarrow \pi_{i}(B)$ is an epimorphism mod finite abelian groups for all odd $i$ sucb that $1<i \leq 4 N+1$.

As an example, let $F$ be a finite dimensional $C W$ complex with $\pi_{*}(F)$ of finite type. Then if $\pi_{3}(F)$ is a finite group we have $G_{2}(F), G_{4}(F), G_{6}(F)$ and $G_{8}(F)$ as finite subgroups. Also, by Weingram's theorem [20], we know that $G_{2}(F)=0$.

\section{BIBLIOGR APHY}

1. Guy Allaud, On the classification of fibre spaces, Math. Z. 92 (1966), 110-125. MR $32 \# 6462$.

2. A. Borel, Topics in the homology theory of fibre bundles, Lecture Notes in Math., no. 36, Springer-Verlag, Berlin and New York, 1967. MR $36 \# 4559$.

3. R. F. Brown, On the Lefshetz number and the Euler class, Trans. Amer. Math. Soc. 118 (1965), 174-179. MR 30 \#3481.

4. Albrecht Dold and René Thom, Quasifaserungen und unendliche symmetrische produkte, Ann. of Math. (2) 67 (1958), 239-281. MR 20 \#3542.

5. James Dugundji, Topology, Allyn and Bacon, Boston, Mass., 1966. MR 33 \#1824.

6. E. Fadell, Generalized normal bundles for locally-flat imbeddings, Trans. Amer. Math. Soc. 114 (1965), 488-513. MR 31 \#4037.

7. Daniel H. Gottlieb, On fibre spaces and the evaluation map, Ann. of Math. (2) 87 (1968), 42-55. MR $36 \# 4560$. 755.

8. —_, Evaluation subgroups of homotopy groups, Amer. J. Math. 91 (1969), 729-

9. - The evaluation map and homology, Michigan Math. J. (to appear). $\# 6630$.

10. I. M. James, On the space of bundle maps, Topology 2 (1963), 45-59. MR 33

11. John Milnor, Construction of universal bundles. II, Ann. of Math. (2) 63 (1956), 430-436. MR 17, 1120.

12. - Lectures on characteristic classes, Princeton University, Princeton, N. J., 1957 (mimeographed). 

$\# 8420$.

13. A. Phillips, Submersions of open manifolds, Topology 6 (1967), 171-296. MR 34

14. E. Spanier, Infinite symmetric products, function spaces and duality, Ann. of Math. (2) 69 (1959), 142-198; erratum ibid., 733 (see §13). MR 21 \#3851.

15. - Algebraic topology, McGraw-Hill, New York, 1966. MR 35 \#1007.

16. N. E. Steenrod, Topology of fibre bundles, Princeton Math. Series, vol. 14, Princeton Univ. Press, Princeton, N. J., 1951, 2nd printing, 1957. MR 12, 522.

17. - Milgram's classifying space of a topological group, Topology 7 (1968), 349-368. MR $38 \# 1675$.

18. T. E. Stewart, Lifting group actions in fibre bundles, Ann. of Math. (2) 74 (1961), 192-198. MR 23 \#A3798.

19. René Thom, L'homologie des espaces fonctionnels, Colloque de Topologie Algébrique (Louvain, 1956), Georges Thone, Liége; Masson, Paris, 1957. MR 19, 669.

20. S. Weingram, On the incompressibility of certain maps, Ann. of Math (2) 93 (1971), $476-485$.

DEPARTMENT OF MATHEMATICS, PURDUE UNIVERSITY, LAFAYETTE, INDIANA 47907 